\title{
Age-dependent SMN expression in disease-relevant tissue and implications for SMA treatment
}

\author{
Daniel M. Ramos, ${ }^{1}$ Constantin d'Ydewalle, ${ }^{2}$ Vijayalakshmi Gabbeta, ${ }^{3}$ Amal Dakka, ${ }^{3}$ Stephanie K. Klein, ${ }^{4}$ Daniel A. Norris, ${ }^{4}$ \\ John Matson, ${ }^{4}$ Shannon J. Taylor, ${ }^{5}$ Phillip G. Zaworski, ${ }^{5}$ Thomas W. Prior, ${ }^{6}$ Pamela J. Snyder, ${ }^{7}$ David Valdivia, ${ }^{2}$ Christine L. Hatem, ${ }^{2}$ \\ Ian Waters, ${ }^{8}$ Nikhil Gupte, ${ }^{9}$ Kathryn J. Swoboda, ${ }^{10}$ Frank Rigo, ${ }^{4}$ C. Frank Bennett, ${ }^{4}$ Nikolai Naryshkin, ${ }^{3}$ Sergey Paushkin, ${ }^{11}$ \\ Thomas 0. Crawford, ${ }^{2,12}$ and Charlotte J. Sumner ${ }^{1,2}$ \\ DDepartment of Neuroscience and ${ }^{2}$ Department of Neurology, Johns Hopkins University School of Medicine, Baltimore, Maryland, USA. ${ }^{3 P T C}$ Therapeutics, South Plainfield, New Jersey, USA. ${ }^{4}$ Ionis \\ Pharmaceuticals, Carlsbad, California, USA. ${ }^{5}$ PharmOptima, Portage, Michigan, USA. ' Center for Human Cenetics, Case Western Reserve University, Cleveland, Ohio, USA. Department of Pathology, \\ Ohio State University, Columbus, Ohio, USA. ${ }^{8}$ The Sidney Kimmel Comprehensive Cancer Center, Department of Oncology, and ${ }^{9}$ Department of Medicine, Johns Hopkins University School of Medicine, \\ Baltimore, Maryland, USA. ${ }^{10}$ Center for Genomic Medicine, Department of Neurology, Massachusetts General Hospital, Harvard Medical School, Boston, Massachusetts, USA. "'SMA Foundation, New York, \\ New York, USA. ${ }^{2}$ Department of Pediatrics, Johns Hopkins University School of Medicine, Baltimore, Maryland, USA
}

BACKCROUND. Spinal muscular atrophy (SMA) is caused by deficient expression of survival motor neuron (SMN) protein. New SMN-enhancing therapeutics are associated with variable clinical benefits. Limited knowledge of baseline and druginduced SMN levels in disease-relevant tissues hinders efforts to optimize these treatments.

METHODS. SMN mRNA and protein levels were quantified in human tissues isolated during expedited autopsies.

RESULTS. SMN protein expression varied broadly among prenatal control spinal cord samples, but was restricted at relatively low levels in controls and SMA patients after 3 months of life. A 2.3-fold perinatal decrease in median SMN protein levels was not paralleled by comparable changes in SMN mRNA. In tissues isolated from nusinersen-treated SMA patients, antisense oligonucleotide (ASO) concentration and full-length (exon 7 including) SMN2 (SMN2-FL) mRNA level increases were highest in lumbar and thoracic spinal cord. An increased number of cells showed SMN immunolabeling in spinal cord of treated patients, but was not associated with an increase in whole-tissue SMN protein levels.

CONCLUSIONS. A normally occurring perinatal decrease in whole-tissue SMN protein levels supports efforts to initiate SMN-inducing therapies as soon after birth as possible. Limited ASO distribution to rostral spinal and brain regions in some patients likely limits clinical response of motor units in these regions for those patients. These results have important implications for optimizing treatment of SMA patients and warrant further investigations to enhance bioavailability of intrathecally administered ASOs.

FUNDING. SMA Foundation, SMART, NIH (R01-NS096770, R01-NS062869), lonis Pharmaceuticals, and PTC Therapeutics. Biogen provided support for absolute real-time RT-PCR.

\section{Introduction}

The neuromuscular disease spinal muscular atrophy (SMA), affecting approximately 1 in 10,000 individuals, is the most common inherited cause of infant death (1). The most frequent and severe type I form causes profound muscle hypotrophy and weak-

Conflict of interest: VG, AD, and NN are employees of PTC Therapeutics. SKK, DAN, JM, FR, and CFB are employees of lonis Pharmaceuticals. CFB holds a patent covering nusinersen licensed to Biogen (8980853). KJS receives grant support from Biogen and Cure SMA. CJS served as a paid advisor, consultant, and/or speaker to the SMA Foundation, Biogen, Ionis Pharmaceuticals, PTC Therapeutics, Roche/Genentech, AveXis, Cytokinetics, and Pfizer and is an associate editor for the JCI. This arrangement has been reviewed and approved by the Johns Hopkins University in accordance with its conflict of interest policies.

Copyright: @ 2019, American Society for Clinical Investigation

Submitted: August 10, 2018; Accepted: August 7, 2019; Published: October 7, 2019

Reference information: / Clin Invest. 2019;129(11):4817-4831.

https://doi.org/10.1172/JCl124120. ness, particularly of bulbar, truncal, and proximal limb muscles. Without treatment, such infants never attain motor milestones, including the ability to sit or stand, and succumb to respiratory insufficiency in the first year or two of life. SMA is caused by recessive, loss-of-function mutations of the survival motor neuron 1 gene (SMN1) (2). All patients retain at least one copy of the paralog gene $S M N 2$, but transcripts arising from SMN2 mostly lack exon 7 and make a truncated, rapidly degraded protein (3-6). A minority of SMN2 transcripts retain exon 7 and encode sufficient full-length SMN protein to enable viability, but insufficient levels to prevent motor neuron degeneration. SMN is ubiquitously expressed and best known for regulating assembly of small nuclear ribonucleoproteins (7). Why and how impairment of this or other putative functions of SMN predominately affects motor neurons are poorly understood. Variation in genomic SMN2 copy number correlates inversely with SMA disease severity $(8,9)$. Most of the severely affected infants inherit 2 copies of SMN2, while those with milder 


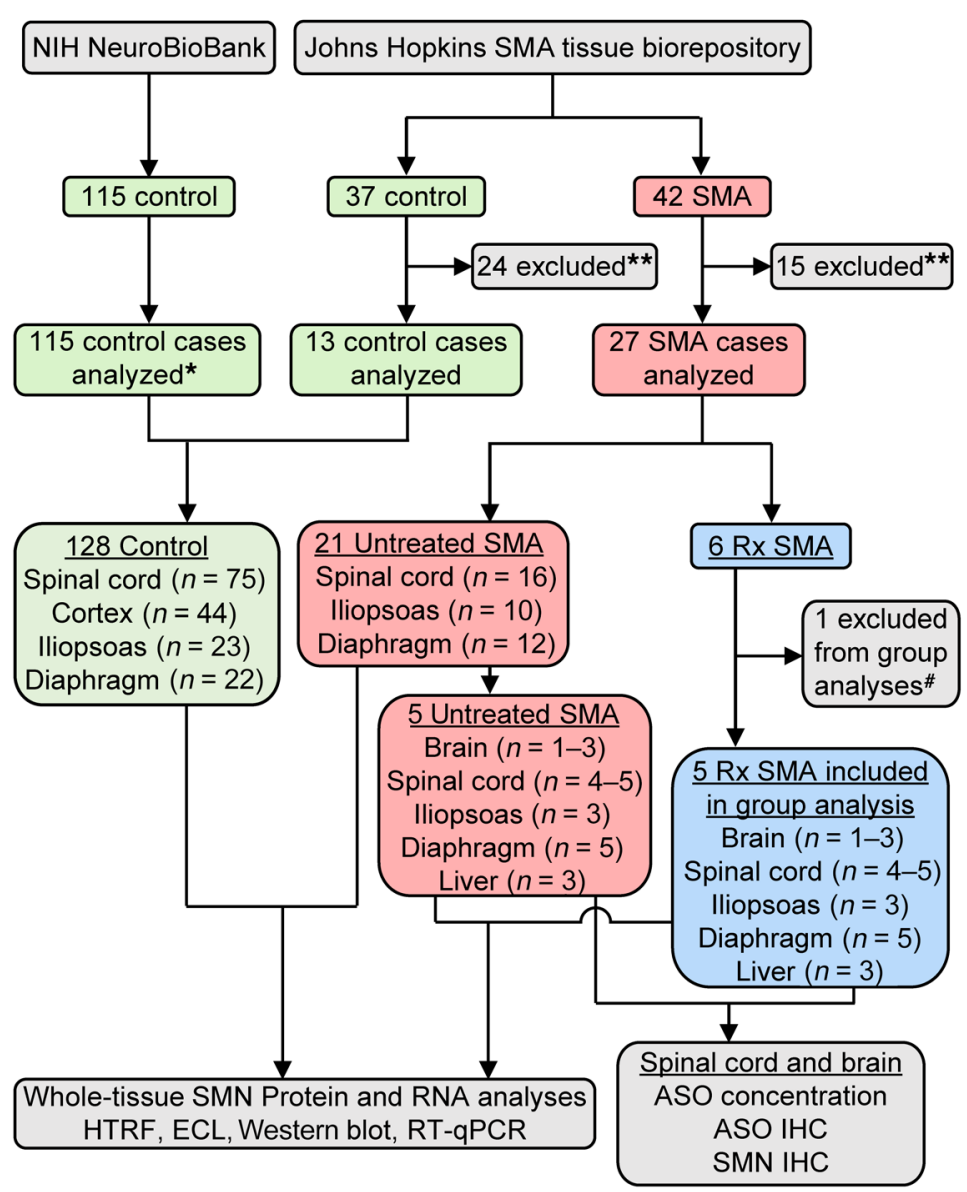

forms have 3 or 4 copies; a few individuals with 5 copies of SMN2 have been asymptomatic (10).

The last decade has witnessed remarkable progress in the development of therapeutic strategies to increase SMN expression in the CNS of SMA patients (11), either by modifying the splicing of SMN2 pre-mRNAs using antisense oligonucleotides (ASOs) (12) or small molecules $(13,14)$ or by delivering exogenous SMN1 using viral vectors (15). Both nusinersen, a splice-switching ASO delivered by lumbar intrathecal injection 4 times in the first 2 months followed by chronic dosing every 4 months, and onasemnogene abeparvovec-xioi, a recombinant self-complementary adeno-associated virus 9 expressing SMN1 cDNA (scAAV9-SMN) delivered once intravenously, were recently shown to improve survival and motor function in infantile SMA patients $(16,17)$. Nusinersen also improves motor function in children and adolescents with milder forms of SMA (18). These results led to rapid FDA approval of nusinersen for SMA patients of all ages in December of 2016. The FDA approved onasemnogene abeparvovec-xioi in May 2019 for infants 2 years and younger. Importantly, however, the trial results demonstrate a spectrum of clinical efficacy. Treated infants manifest a range of benefit ranging from attainment of major motor milestones to little or no change in motor function. One factor that appears to play a key role in therapeutic efficacy is earlier age at treatment initiation, but mechanisms that underlie this temporal dependency in humans have not been determined. While the goal of each of these therapies is to increase SMN expression in
Figure 1. Flow diagram outlining study design and patient samples included. * Seventeen cases had chromosomal abnormalities and/or CNS malformations. ${ }^{*}$ Relevant tissues not available. "Blood observed in CSF sample at time of final nusinersen injection.

motor neurons, there are few data about baseline normal or disease-associated SMN levels in disease-relevant human tissues and there is even less understanding of either changes in SMN expression over the course of development or of changes with treatment because SMN levels cannot be measured in the CNS of living patients. Understanding the dynamics of SMN expression in the human CNS and non-CNS tissues of unaffected and SMA patients will enable further optimization of SMNaugmenting treatments.

\section{Results}

We implemented an expedited autopsy protocol to dissect and immediately freeze tissues isolated from 37 non-SMA control subjects and 42 SMA patients of different ages. Of these, 13 control and 27 SMA cases were analyzed, along with 115 control cases from the NIH NeuroBioBank (Figure 1); 38\% of cases had a postmortem interval (PMI) of 6 or fewer hours, $68 \%$ of 12 or fewer hours, and $97 \%$ of 24 or fewer hours. All control cases had at least 1 copy of SMN1. All SMA cases, except the one fetal SMA sample, had O SMN1 copies and 2 SMN2 copies, consistent with their early age of disease onset (fetal sample: SMN2 copy number = 3) (Supplemental Table 1; supplemental material available online with this article; https:// doi.org/10.1172/JCI124120DS1).

Spinal cord SMN protein levels are high during fetal development and decrease during the perinatal period. SMN protein levels were measured in 91 thoracic or lumbar spinal cord samples ( 75 control, 16 SMA; the one fetal SMA sample was from an unspecified spinal level) isolated from subjects ranging in age from 15 weeks gestation to 14 years by homogenous time-resolved fluorescence (HTRF) (Figure 2A), a fluorescence resonance energy transfer-based (FRET-based) technology often used in high-throughput screening (19). SMN protein levels varied widely, ranging in expression from $27 \Delta \mathrm{F} / \mathrm{mg}$ to $2288 \Delta \mathrm{F} / \mathrm{mg}$ in control samples, but median SMN protein was 2.3-fold higher in prenatal controls compared with postnatal controls younger than 3 months (defined as early postnatal) and 6.5-fold higher than in postnatal controls aged 3 months through 14 years (defined as late postnatal) (Figure 2B). The decrease in SMN expression was most evident in samples spanning the 3 months before and 3 months after birth (the perinatal period). Thereafter, SMN levels remained low in cases aged 3 months through 14 years. In some control cases, fetal death was caused by chromosomal abnormalities and/or CNS malformations (Figure 2A), and those samples had lower SMN levels (median: $1004 \Delta \mathrm{F} / \mathrm{mg}$ in prenatal control samples without and $195 \Delta \mathrm{F} / \mathrm{mg}$ with chromosomal abnormalities and/or CNS malformations, $P=0.0014$ ). A sensitivity analysis indicated that inclusion of these samples in our statistical analyses did not change outcomes (data not shown). 

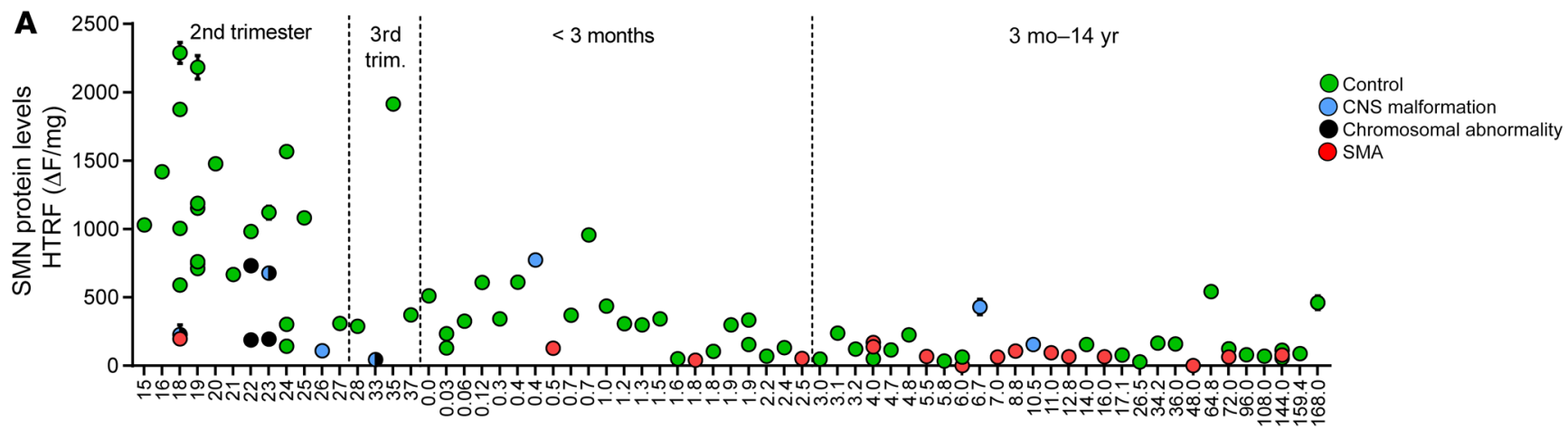

ـ GA (weeks) _ــــ Age (months)
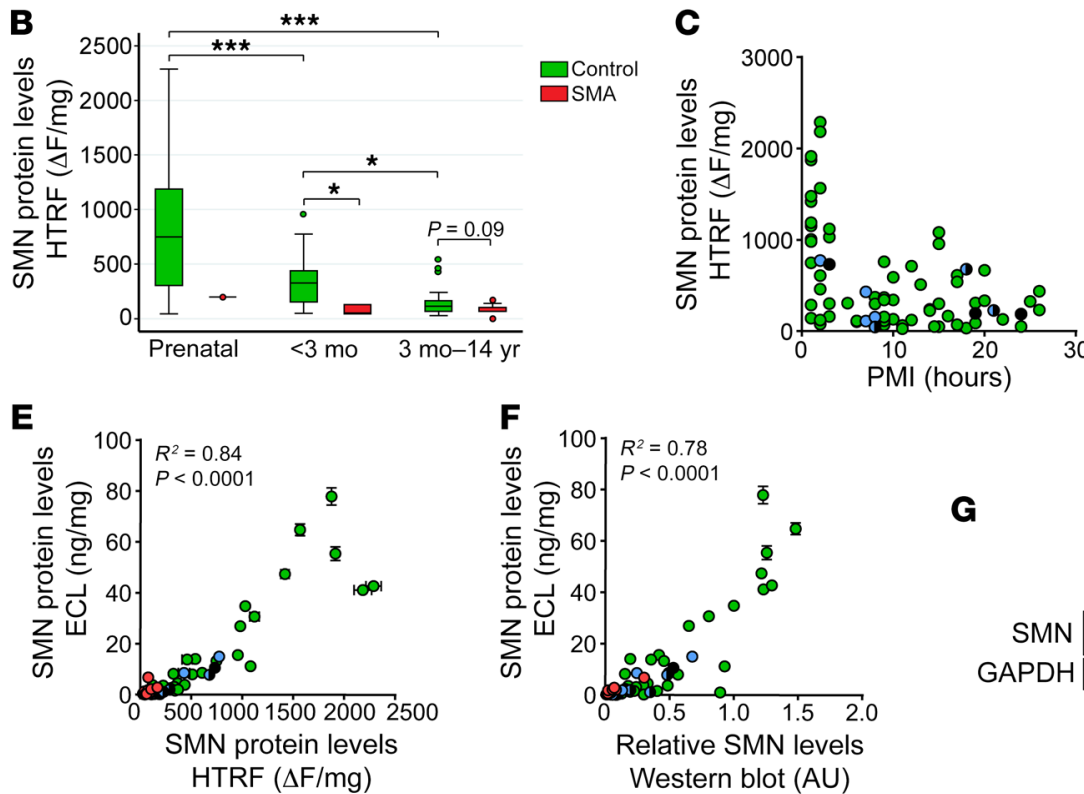

H

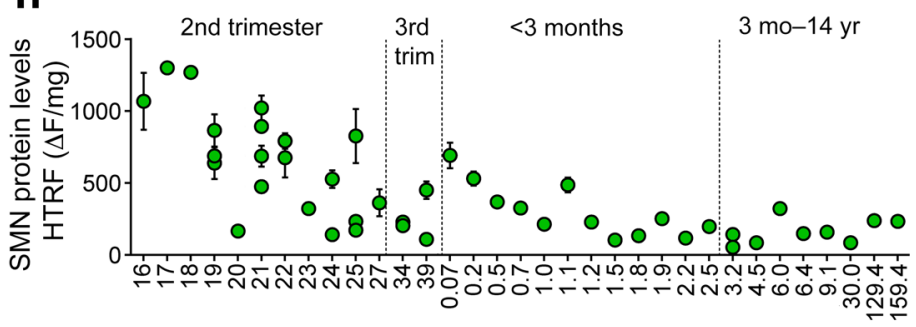

GA (weeks)

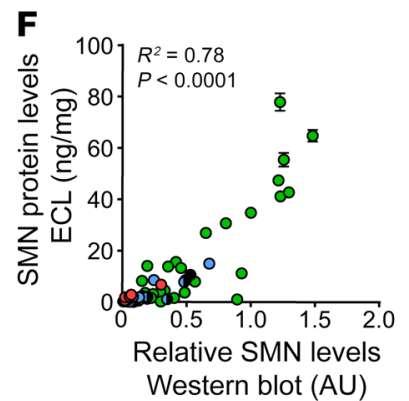

I

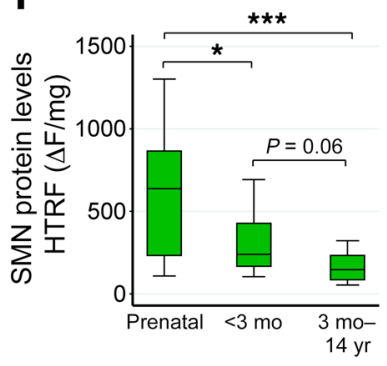

D

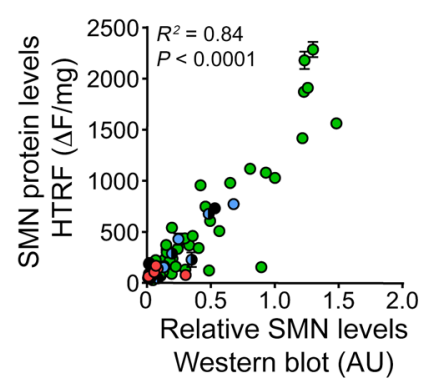

G GA (weeks) Age (months)
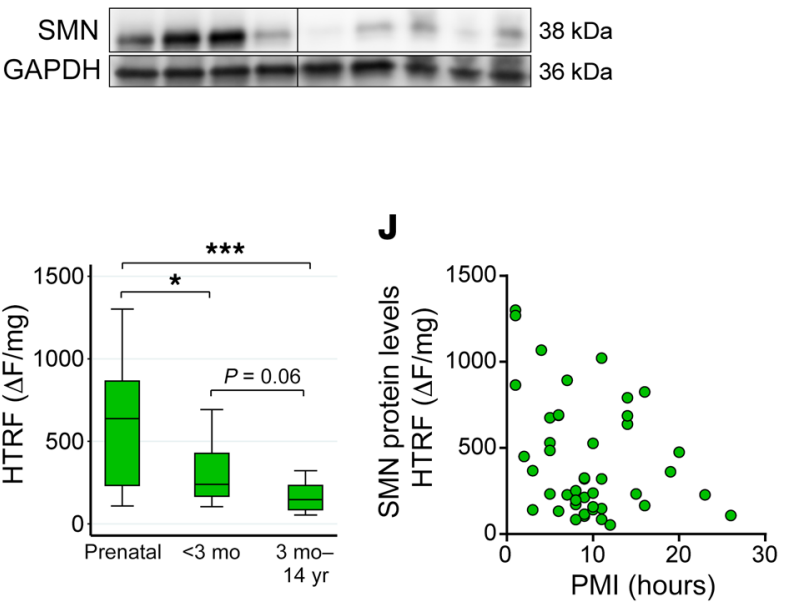

Figure 2. Spinal cord and cortex SMN protein levels are higher in fetal samples than in postnatal samples. (A) SMN protein expression quantified by HTRF in spinal cord samples ( $n=75$ control; $n=16$ SMA patients) ranging in age from 15 weeks gestation (GA) to 168 months. (B) Medians, interquartile range (box), and 95th percentiles (whiskers) of data from A. (C) Scatter blot of PMI against SMN protein expression in control samples. (D-F) Correlations between SMN protein measured by (D) HTRF and Western blot, (E) ECL and HTRF, and (F) ECL and Western blot. (C) Representative Western blot of SMN expression in prenatal and postnatal control samples. All lanes were run on a single gel. Black line indicates discontinuous lanes. (H) SMN protein expression quantified by HTRF in cortex samples ( $n=44$ control) ranging in age from 16 weeks gestation to 159.4 months. (I) Medians, interquartile range (box), and 95th percentiles (whiskers) of data from $\mathbf{H}$. (J) Scatter plot of PMI against SMN protein expression in all control cortex samples. SMN molecular weight $=38 \mathrm{kDa}$; GAPDH molecular weight $=36 \mathrm{kDa} .{ }^{*} P<0.05 ;{ }^{* * *} P<0.001$, representing statistical analysis performed before multiple comparisons. $P$ values of pairwise comparison of medians were calculated using Wilcoxon's rank-sum test. Prenatal SMA group excluded in all statistical analyses. Adjustment for multiple comparisons was applied to control groups between time points. The $\alpha$ level was set to $0.0167(0.05 / 3)$ using Bonferroni's adjustment.

In SMA spinal cord tissues, SMN protein levels were low in all postnatal cases assessed (range: 0.5 months-12 years). SMN protein expression was approximately 6-fold lower in SMA spinal cord samples compared with control samples aged 0-3 months, but only trended toward being decreased in samples greater than 3 months (Figure 2B), suggesting that differences in SMN levels between control and SMA diminish with increasing age. The single fetal SMA case (18 weeks gestational age [GA]) 
A

A

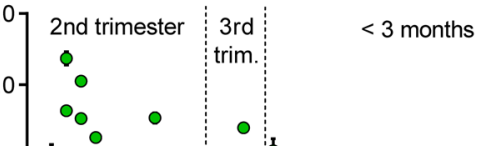

2nd trimester 3 ard $\quad<3$ months

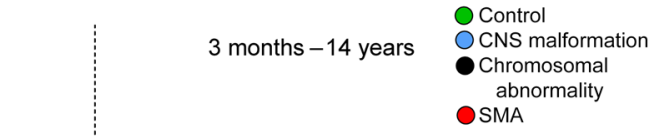

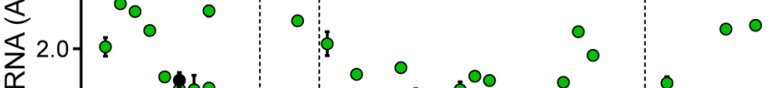

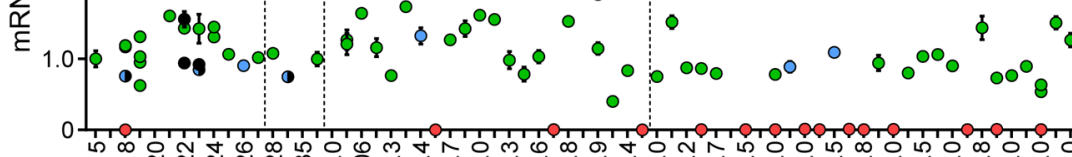

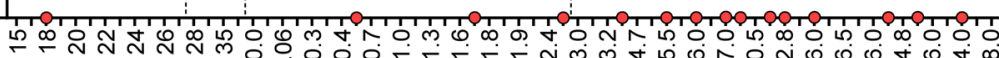

$\llcorner\mathrm{GA}$ (weeks)

Age (months)

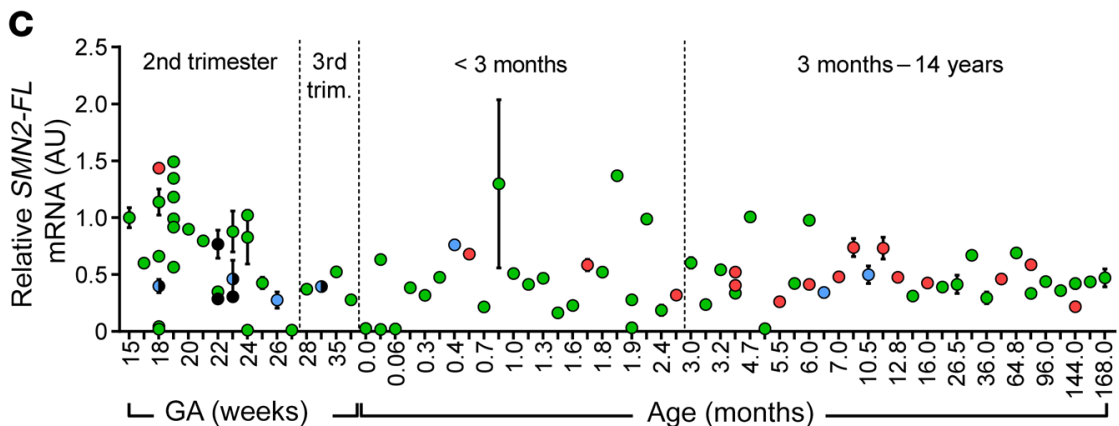

ᄂ GA (weeks) Age (months)

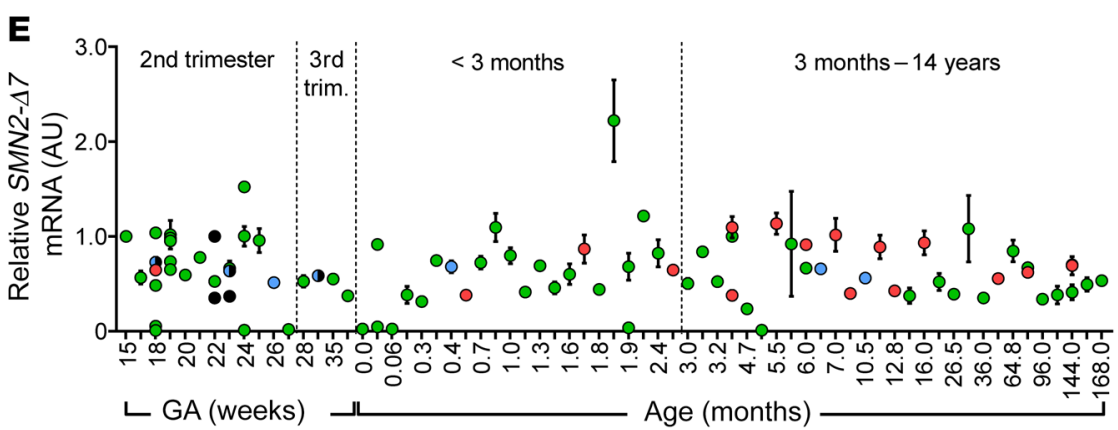

G

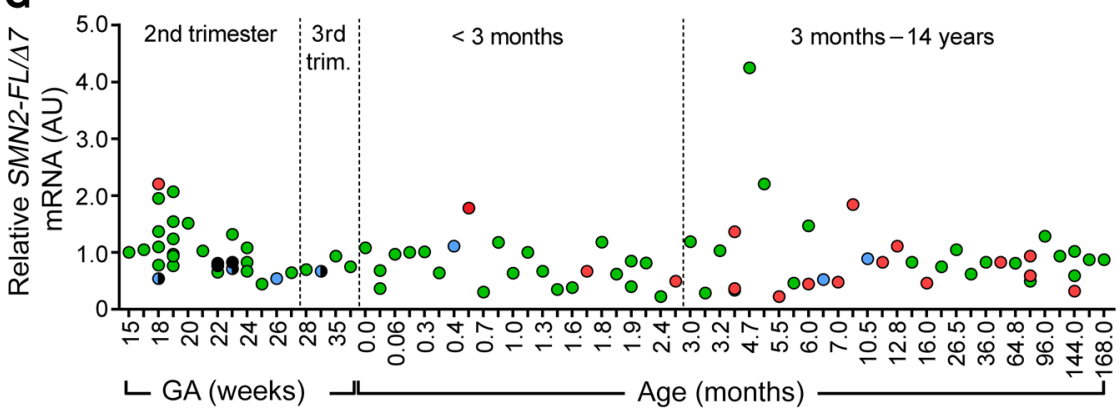

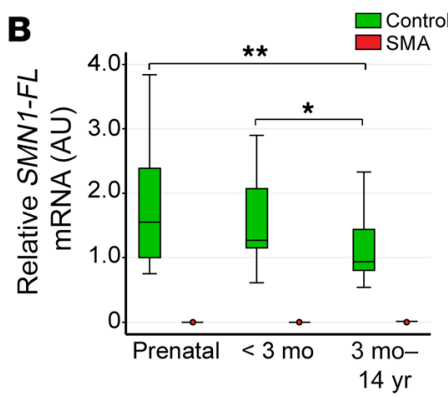

D
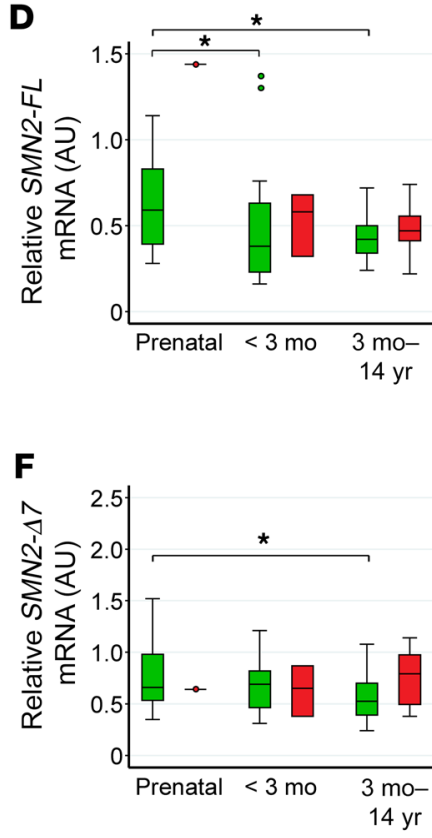

H

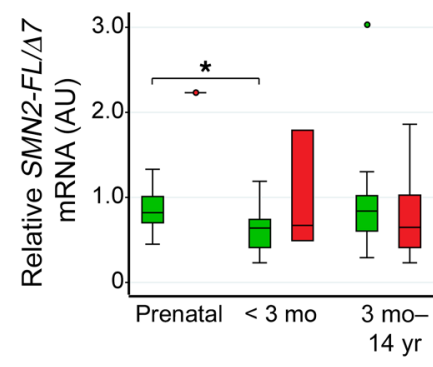

Figure 3. SMN mRNA levels decrease in human thoracic spinal cord cases of different ages. (A-H) Case-by-case (A) full-length SMN1 (SMN1-FL) mRNA, (C) full-length SMN2 (SMN2-FL) mRNA, (E) truncated SMN2- $\triangle 7$ mRNA, and (C) the ratio of SMN2-FL/A7 mRNA. (B, D, F, and $\mathbf{H})$ Medians, interquartile range, and 95 th percentiles (whiskers) for data in (A, C, E, and $\mathbf{C}$ ), respectively. ${ }^{*} P<0.05$; ${ }^{*} P<0.01$, representing the statistical analysis performed before multiple comparisons ( $n=75$ control, $n=16 \mathrm{SMA}$ ). $P$ values of pairwise comparison of medians were calculated using Wilcoxon's rank-sum test. Prenatal SMA group excluded in all statistical analyses. Adjustment for multiple comparisons was applied to control groups between time points. The $\alpha$ level was set to $0.0167(0.05 / 3)$ using Bonferroni's adjustment.

had the highest SMN expression of SMA cases (SMA prenatal: $198 \Delta \mathrm{F} / \mathrm{mg}$ vs. postnatal SMA median: $66 \Delta \mathrm{F} / \mathrm{mg}$; Figure 2B), but was approximately 5 -fold reduced compared with median SMN levels of prenatal control samples. No differences were seen in median SMN levels between early postnatal and late postnatal SMA samples. When Bonferroni's correction for multiple comparisons between control cases in the 3 age groups was applied, threshold for significance $(\alpha)$ was adjusted to 0.0167 and all associations remained significant.

To further validate the data obtained with HTRF, we quantified SMN protein levels using 2 other methods: electrochemiluminescence (ECL) (20) and Western blot. Very similar results were obtained with all 3 detection methods, and correlation coefficients between each of the methods were robust (Figure 2, D-F). A rep- 

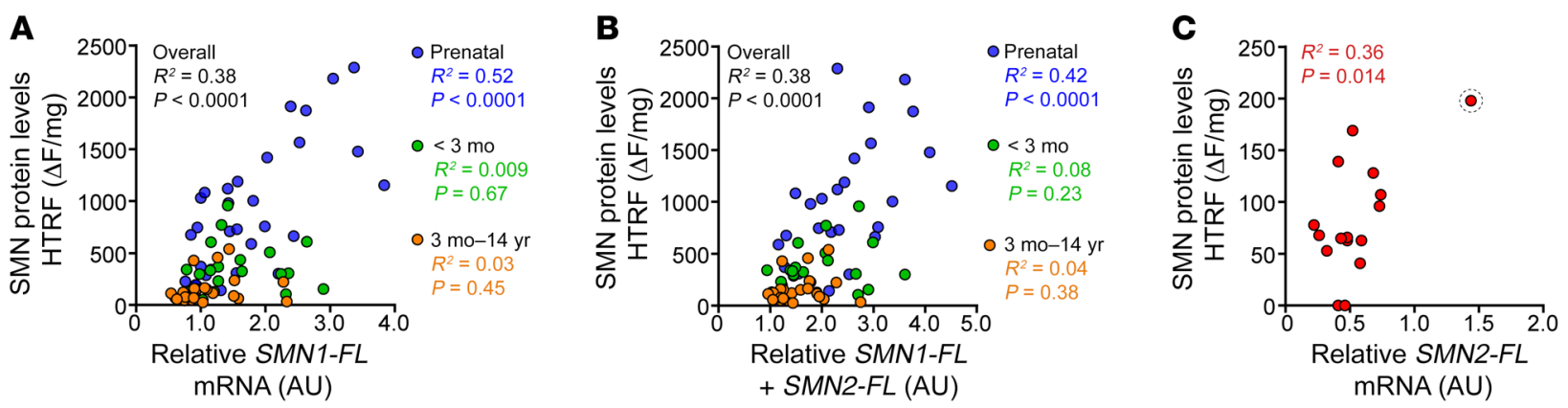

Figure 4. SMN protein expression correlates with SMN1-FL prenatally, but not postnatally, in human spinal cord. (A) Correlation between SMN protein measured via HTRF and SMN1-FL mRNA expression in control human thoracic spinal cord cases $(n=75)$ stratified by indicated age bins. (B) Correlation of SMN protein with the sum of SMN1-FL+SMN2-FL mRNA. (C) Correlation between SMN protein measured via HTRF and SMN2-FL mRNA in SMA cases ( $n=$ 16). Single prenatal SMA case circled. Linear regression analysis was performed to achieve $R^{2}$ and $P$ values.

resentative Western blot of prenatal and postnatal control samples is shown in Figure 2G (full uncut gel available in supplemental material). There was also strong concordance between protein extractions done on 2 independent thoracic spinal cord tissue samples for each of 3 controls and 3 SMA cases (data not shown).

To assess the possible impact of PMI, we first plotted SMN protein levels against PMI on a scatterplot (Figure 2C). Based on the distribution of the data, we then modeled PMI with a quadratic term for univariable quantile regression analysis $(P<0.01)$. Multivariable quantile regression indicated that SMN protein significantly decreased with age after adjusting for PMI (prenatal vs. 0-3 months, $P=0.002$; prenatal vs. 3 months -14 years, $P<0.001)$. To further examine the possible impact of PMI, we also assessed a separate cohort of brain frontal cortex samples from control subjects of different ages for which tissues with more optimally matched PMI were available. SMN protein expression in prenatal controls was 2.6-fold higher compared with that in early postnatal controls and 4.3-fold higher compared with late postnatal samples, with the largest decrease occurring perinatally (Figure 2, H and I). After Bonferroni's correction for multiple comparisons, the significance between prenatal and early postnatal controls was slightly attenuated (Bonferroni's adjusted $\alpha=0.0167$ compared with $P$ value of 0.02). This may be a function of the relatively small data set size. We plotted SMN protein against PMI on a scatterplot (Figure 2J) and modeled PMI using a quadratic term for univariable quantile regression analysis, which indicated that PMI did not significantly affect the decrease of SMN protein with age $(P=0.33)$.

Spinal cord SMN mRNA expression correlates moderately with protein expression. SMN mRNA levels were measured using realtime reverse-transcription quantitative PCR (RT-PCR) and TaqMan assays specific for full-length (exon 7 including) SMN1 mRNA (SMN1-FL), full-length SMN2 (SMN2-FL), and SMN2 mRNA lacking exon 7 (SMN2-47) (Supplemental Table 2 and ref. 21). As expected, all control spinal cord samples expressed SMN1-FL transcript and SMA samples did not. Also as expected, the control samples with 0 copies of SMN2 did not express SMN2 transcripts, and thus these samples were not included in median calculations (Figure 3, C-H, and Supplemental Figure 1, B-D). Overall, the range of SMN transcript levels was restricted compared with that of protein and the decreases in median SMN1-FL or SMN2-FL mRNA levels between prenatal and postnatal sam- ples (SMN1-FL, 25\%; SMN2-FL, 29\%) were modest compared with those of protein (79\%). Interestingly, in both spinal cord and cortex samples, a significant drop of median SMN2-FL transcript occurred only between prenatal and early postnatal control samples (Figure 3, C and D, and Supplemental Figure 1B), whereas median SMN1-FL mRNA levels decreased significantly only between early and late postnatal samples (Figure 3, A and B, Supplemental Figure 1A, and Supplemental Tables 3 and 4). This may indicate an earlier developmental decrease in $S M N 2$ expression relative to that of SMN1. SMN2- 47 mRNA levels and SMN2$F L / \Delta 7$ ratios changed minimally between prenatal and postnatal controls, indicating little effect of age on exon 7-splicing patterns (Figure 3, E-H, Supplemental Figure 1, C and D, and Supplemental Tables 3 and 4). Either very weak or no correlation was observed between SMN transcript expression and PMI in spinal cord or cortex samples (Supplemental Figure 2). As some $S M N$-derived transcripts may lack exon $5(22,23)$, we also examined levels of $S M N$ mRNA lacking exon $5(S M N-\triangle 5)$ in a subset of 4 prenatal and 5 postnatal control samples. Although detectable in all samples, no differences were seen between prenatal and postnatal samples (data not shown).

To determine the relationships between SMN mRNA and protein levels, we examined case-by-case correlations (Figure 4). When including all control samples across the age spectrum, SMN protein only modestly correlated with $S M N 1-F L$ mRNA levels. Importantly, the correlation between $S M N 1$ mRNA expression and SMN protein was driven by prenatal samples (Figure $4 \mathrm{~A}$ ). The sum of SMN1-FL and SMN2-FL levels showed similar results (Figure $4 \mathrm{~B})$. In SMA cases, SMN protein modestly correlated with SMN2$F L$ levels. Of note, the single prenatal SMA case expressed the highest SMN protein and SMN2-FL levels (Figure 4C). We applied a multivariable quantile regression analysis to determine whether changes in SMN1-FL and SMN2-FL expression could account for the decrease in SMN protein. Although SMN1-FL and SMN2-FL levels significantly contributed to SMN protein expression (SMN1$F L, P<0.001$; SMN2-FL, $P=0.03$ ), SMN protein independently decreased with age between prenatal and late postnatal samples $(P=0.003)$ after adjusting for SMN1-FL and SMN2-FL expression and PMI. This may indicate that additional, posttranscriptional mechanisms contribute to the decrease in SMN protein levels during perinatal development. Comprehensive tables of protein 

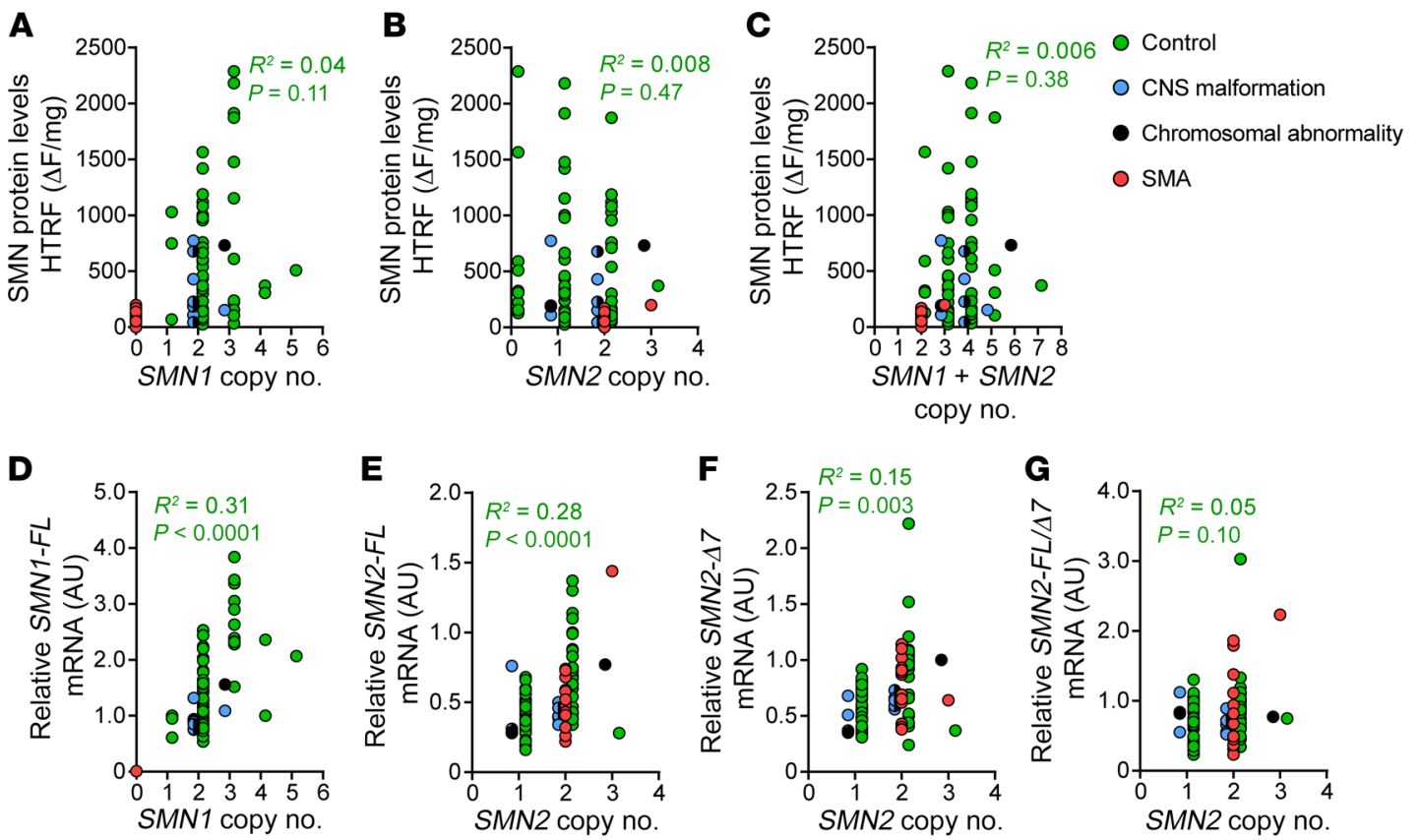

Figure 5. SMN1 and SMN2 copy numbers correlate poorly with SMN protein expression. (A-C) Correlation between SMN protein levels in control ( $n=75$ ) thoracic spinal cords and (A) SMN1 copy number, (B) SMN2 copy number, or (C) the sum of SMN1+SMN2 copy number. (D) SMN1 copy number correlation with SMN1-FL mRNA. (E-C) SMN2 copy number correlation with (E) SMN2-FL, (F) SMN2- $\triangle 7$, or (C) ratio of SMN2-FL/A7 mRNA. Linear regression analysis was performed to achieve $R^{2}$ and $P$ values. SMA cases $(n=16)$ excluded in analysis.

and RNA comparisons are available for spinal cord (Supplemental Table 3) and cortex (Supplemental Table 4).

We next determined whether SMN1 and SMN2 gene copy numbers were important determinants of SMN protein and mRNA levels in control spinal cord samples, excluding cases with chromosomal abnormalities and CNS malformations (Figure 5) and cortex samples (Supplemental Figure 3). Modest correlations were observed between SMN1 copy number and SMN1 transcript levels (Figure 5D) as well as SMN2 copy number and SMN2-FL transcript levels (Figure 5E), but not between SMN2 copy number and $S M N 2-\Delta 7$ transcript levels or SMN2-FL/ 47 ratio (Figure 5, F and G). Neither SMN1 copy number, SMN2 copy number, nor the sum of SMN1 and SMN2 copy numbers significantly correlated with SMN protein levels in controls (Figure 5, A-C). When controlled for copy number, SMN2-FL, SMN2- 4 7, and SMN2-FL/ 47 transcript expression did not significantly differ between postnatal control (2 SMN1 copies, 2 SMN2 copies) and postnatal SMA (O SMN1 copies, 2 SMN2 copies) spinal cord or cortex samples (data not shown), suggesting that $S M N 2$ transcript expression is unaltered by the loss of $S M N 1$ in the CNS. Although not reaching statistical significance, when controlled for SMN2 copy number, postnatal control cases had median 2.1-fold more SMN protein than postnatal SMA cases (median control: $143 \Delta \mathrm{F} / \mathrm{mg}$ vs. SMA: $67 \Delta \mathrm{F} / \mathrm{mg} ; P=0.061)$.

Similar developmental SMN expression patterns are observed in skeletal muscle. While spinal cord is considered the principal site of pathology in SMA, SMN-deficient muscle may also play an important role in disease manifestations $(24,25)$. We therefore assessed SMN levels in clinically affected iliopsoas muscles isolated from 14 prenatal controls, 9 postnatal controls, and 10 postnatal SMA cases as well as in clinically resistant diaphragm muscles isolated from 16 prenatal controls, 6 postnatal controls, and 12 postnatal SMA cases (Supplemental Table 1). The median SMN protein levels measured by HTRF (Figure 6A), ECL, and Western blot (not shown) were similar in postnatal muscle and CNS tissues. Similarly to patterns found in the CNS, SMN protein levels decreased approximately 3-fold in postnatal compared with prenatal control iliopsoas samples. However, prenatal diaphragm SMN protein levels were 2-fold less than prenatal iliopsoas muscle samples $(P=0.003)$. SMN protein levels were reduced approximately 2 -fold in postnatal SMA iliopsoas and diaphragm compared with postnatal control samples. SMN transcript levels were also similar across tissues (Figure 6, B-E), with the exception of the muscle tissues in which SMN1 was 2- to 3-fold higher relative to CNS tissues both prenatally and postnatally (Figure 6, B-E). Comprehensive comparisons between tissues are available in Supplemental Tables 5 and 6.

Intrathecal nusinersen treatment results in variable SMN induction in the CNS. To determine the impact of treatment on SMN mRNA and protein levels, we examined tissues isolated from 5 SMA patients who had been treated with nusinersen (number of doses received ranging from 1 to 11) as well as tissues isolated from 5 age-matched, untreated SMA patients (Supplemental Table 1). In treated cases, ASO drug concentrations ranged from $6.74 \mu \mathrm{g} / \mathrm{g}$ to $27.69 \mu \mathrm{g} / \mathrm{g}$ in lumbar and thoracic spinal cord regions; 2-fold lower levels were seen in cervical spinal cord, and variable drug levels were seen in brain tissue for the 3 patients who had available tissue (Figure 7, A and F, Supplemental Figures 4 and 5, and Supplemental Table 7). This pattern of drug concentration was associated with a 3-fold increase in SMN2-FL mRNA levels in cervical, thoracic, and lumbar/sacral spinal cord samples isolated from nusinersen-treated cases (Figure 7, B-F). Although fewer brain 

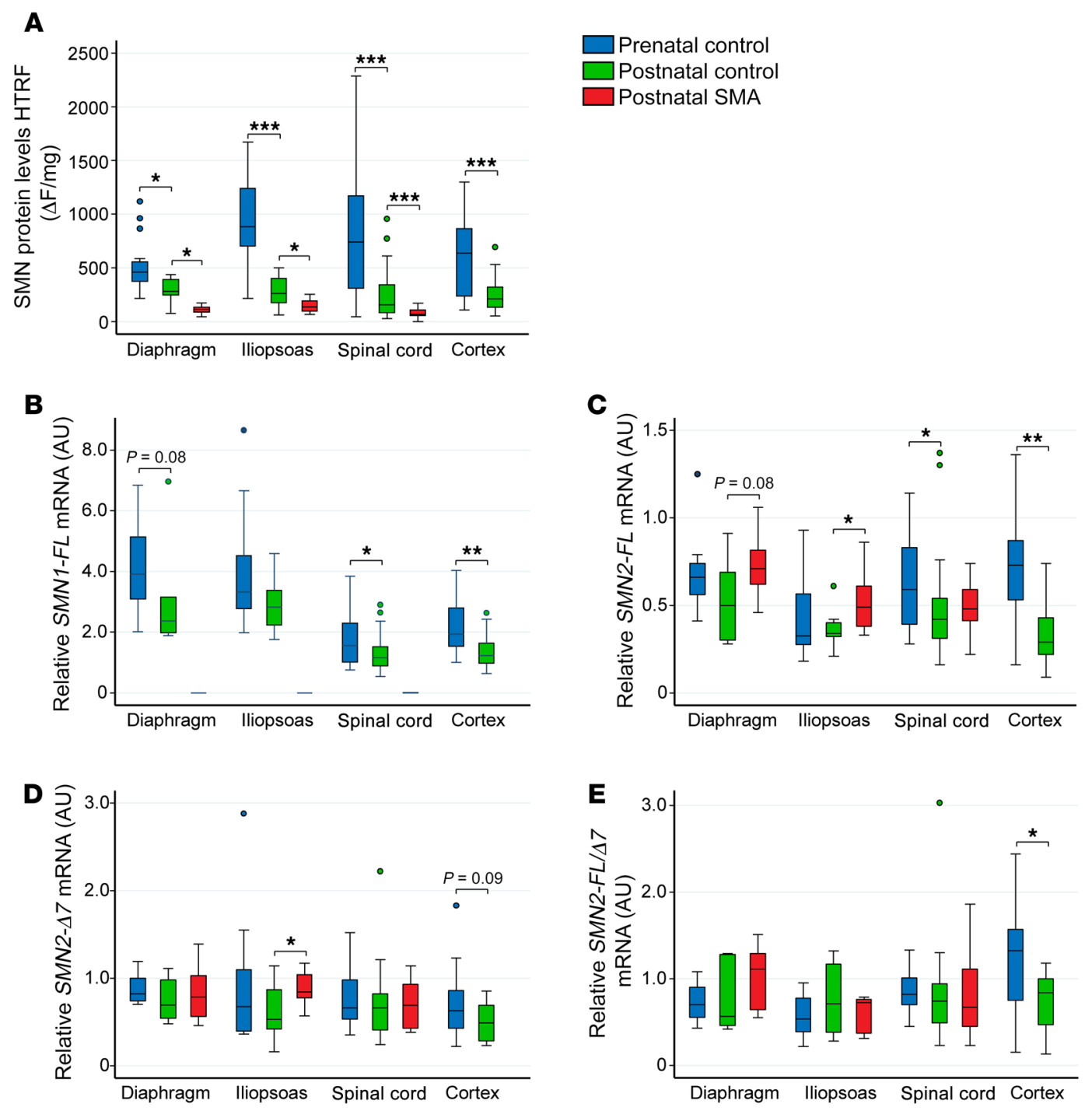

Figure 6. Developmental expression patterns of SMN protein and mRNA are similar in spinal cord, clinically affected iliopsoas muscle, and clinically resistant diaphragm muscle. (A-E) Comparisons of (A) SMN protein measured via HTRF, (B) SMN1-FL, (C) SMN2-FL, (D) SMN2- $\triangle 7$ mRNA expression, or (E) SMN2-FL/A7 ratio between diaphragm, iliopsoas, and spinal cord in prenatal control, postnatal control, and postnatal SMA cases. ( $n$ of diaphragm: $n$ $=16$ prenatal control, $n=6$ postnatal control, $n=12$ postnatal SMA; iliopsoas: $n=14$ prenatal control, $n=9$ postnatal control, $n=10$ postnatal SMA; spinal cord: $n=31$ prenatal control, $n=44$ postnatal control, $n=15$ postnatal SMA; cortex: $n=23$ prenatal control, $n=21$ postnatal control). Data are presented as medians, interquartile range (box), and 95 th percentiles (whiskers). ${ }^{*} P<0.05 ;{ }^{* *} P<0.01 ;{ }^{* *} P<0.001$. Statistical analysis was performed using 2 -way ANOVA and corrected for multiple comparisons. $P$ values of pairwise comparison of medians were calculated using Wilcoxon's rank-sum test.

samples were available ( 2 from cases who had received multiple doses of nusinersen), those that were assessed showed no change in SMN2-FL expression (Figure 7B). As expected, muscle and liver tissues also showed no increase of $S M N 2-F L$ expression. Decreases in SMN2- $\triangle 7$ levels were not robust (Figure 7C), but $S M N 2-F L /$ $\Delta 7$ ratios highlighted a caudal-to-rostral gradient in the CNS that paralleled drug levels (Figure 7D and Supplemental Figure 4A). Changes of SMN2 transcript levels measured by absolute realtime RT-PCR (26) in a subset of samples were highly correlated with those measured by relative RT-qPCR) (Supplemental Figure $4, \mathrm{~B}$ and $\mathrm{C}$ ). The nusinersen-treated case with the least robust increase of the $S M N 2-F L / \Delta 7$ ratio had received a single dose of nusinersen just 5 days before death, whereas the 2 cases with the highest ratios had received multiple doses, with the last given 2 months prior to death (Figure 7F). No increases in whole-tissue SMN protein levels were observed (Figure 7, $\mathrm{E}$ and $\mathrm{F}$ ). In one additional 11-month-old child who received 5 doses of nusinersen (the last 5 days prior to death), drug concentrations in a detailed set of CNS tissues showed a caudal-to-rostral gradient, but little induction of SMN transcript or protein was observed (Supplemental Figure 4, D and E). Importantly, the final dosing was complicated by the presence of blood in the CSF sample, raising doubt about drug delivery, and thus this case was excluded from group analyses.

To further understand the effects of nusinersen, we next assessed ASO and SMN immunostaining in spinal cord sections from the nusinersen-treated patients. Immunohistochemistry using an antibody targeting the nusinersen ASO backbone revealed ASO uptake in a subset of cell types in the spinal cord, 

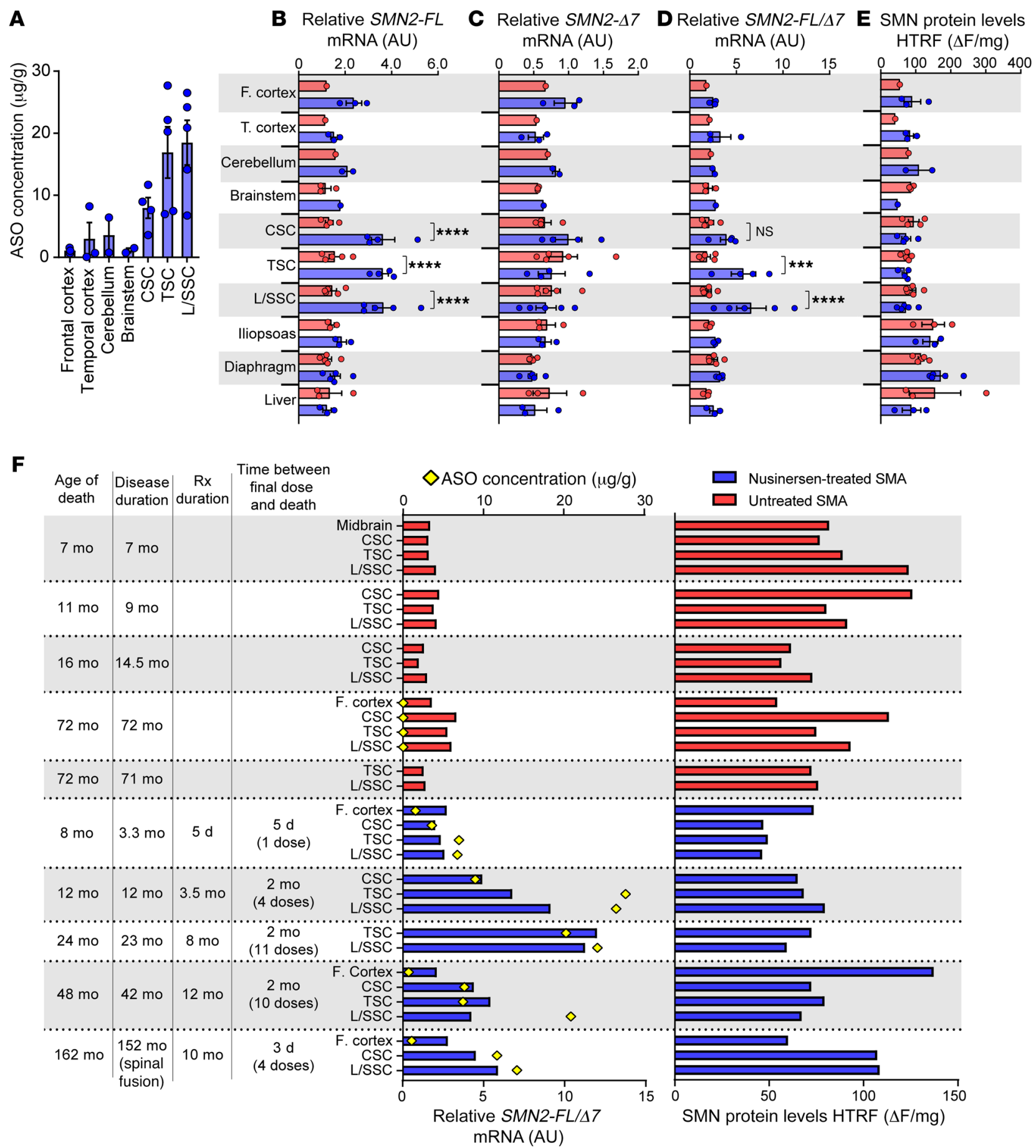

Figure 7. SMN mRNA induction in spinal cord, but not other CNS or peripheral tissues in nusinersen-treated SMA cases. (A) Concentration of ASO quantified from various CNS tissues of nusinersen-treated SMA patients. (B-D) Relative amount of (B) SMN2-FL mRNA, (C) SMN2- $\triangle 7$ mRNA, (D) ratio of SMN2-FL/D7 mRNA in CNS and non-CNS tissues of nusinersen-treated SMA patients ( $n=1-5$, depending on tissue) compared with that of untreated SMA patients ( $n=1-5$, depending on tissue). (E) SMN protein expression measured in whole tissue by HTRF. (F) Case-by-case SMN induction (SMN2-FL/A7) and protein expression in whole spinal cord of nusinersen-treated SMA cases and untreated SMA controls. ASO concentration in each tissue indicated with a yellow diamond. Data are represented as median \pm SEM. ${ }^{* * *} P<0.001 ;{ }^{* * *} P<0.0001$. Statistical analysis was performed using 2-way ANOVA (B, C, D, and $\mathbf{E}$ ) and corrected for multiple comparisons for tissues with $n \geq 3$. Rx, nusinersen treatment; CSC, cervical spinal cord; TSC, thoracic spinal cord; L/SSC, lumbar/sacral spinal cord.

with staining particularly evident in ventral horn neurons. ASO staining intensity was highest in lumbar/sacral and thoracic spinal cord, less in cervical spinal cord (Figure 8, A-C), and modest in upper brain regions (Supplemental Figure 5). SMN immunostain- ing was observed in a small percentage of total spinal cord cells in both control and SMA subjects, and this staining was evident in ventral horn neurons, macroglia, and ependymal cells. To quantify SMN protein expression, all cells were identified, counted, 

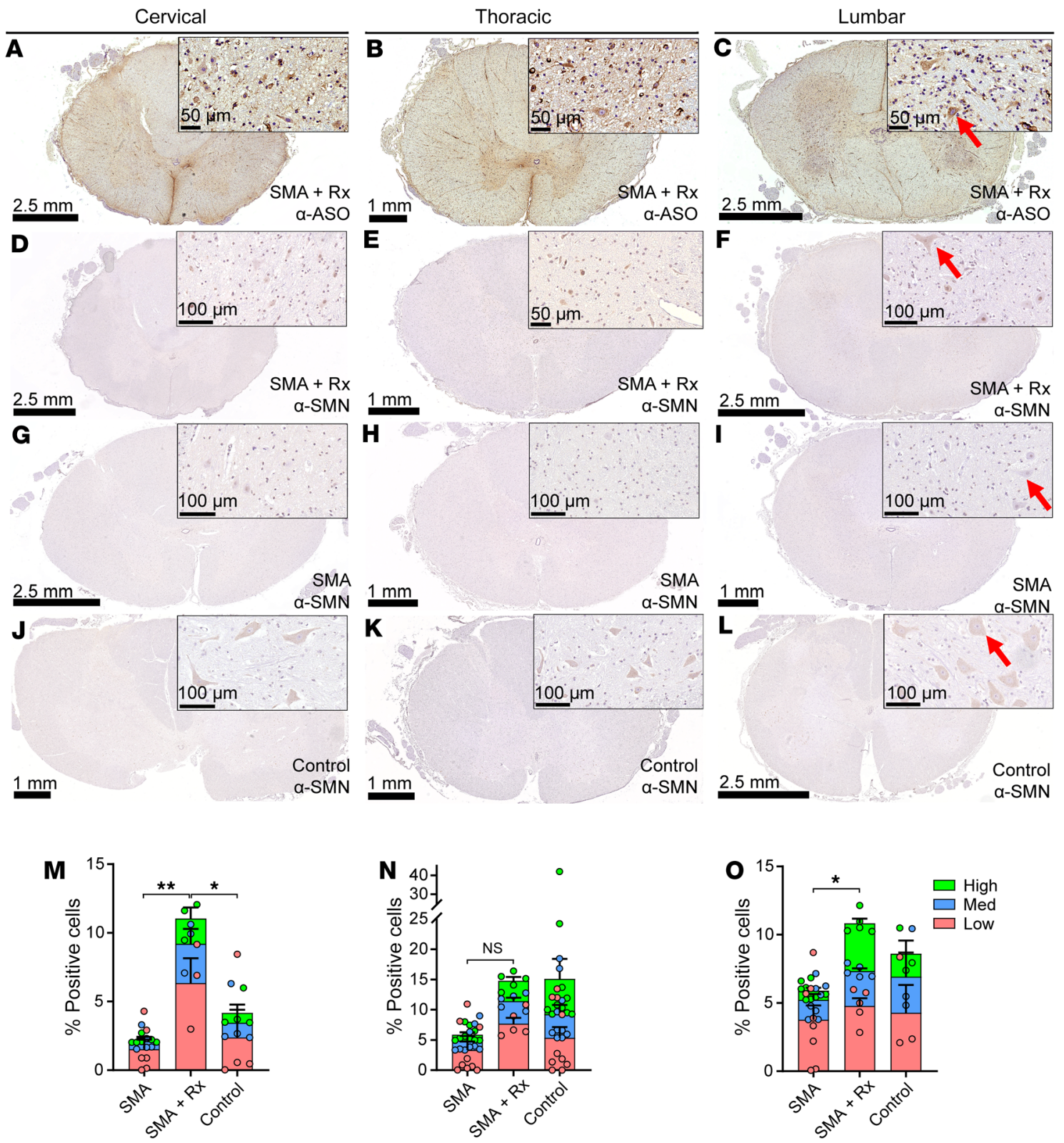

Figure 8. ASO and SMN IHC in nusinersen-treated patient spinal cords. (A-C) IHC for the ASO in (A) cervical, (B) thoracic, and (C) lumbar spinal cord of nusinersen-treated SMA patients. (D-L) IHC for SMN in cervical (D, G, and J), thoracic (E, H, and $\mathbf{K}$ ), and lumbar (F, I, and $\mathbf{L})$ spinal cord of nusinersen-treated SMA patient (D-F), untreated SMA patient (G-I), and untreated control (J-L). High-power images shown in insets. Scale bars are indicated for each image. Arrows depict ventral horn neurons. (M-0) Percentages of cells expressing low-, medium-, or high-intensity level of SMN staining in (M) cervical, (N) thoracic, and (O) lumbar spinal cord of untreated (SMA, $n=6-9$ ), nusinersen-treated controls (SMA + Rx, $n=3-5)$, or untreated controls $(n=3-10)$. Statistical analysis was performed using 2-way ANOVA and corrected for multiple comparisons. ${ }^{*} P<0.05$; ${ }^{* *} P<0.01$. Cervical spinal cord: SMA vs. SMA + Rx (low SMN): $P<0.01$; SMA + Rx vs. control (low SMN): $P<0.05$. Lumbar spinal cord: SMA vs SMA + Rx (high SMN): $P<0.01$.

and SMN expression quantified using Neuron ID 2-RBD software with a user-trained paradigm. Cells were classified as having low, medium, or high SMN staining or undetectable SMN staining (see Methods). In lumbar spinal cord, both the total percentage of SMN-positive cells and the percentage of high-SMN-positive cells in treated patients were significantly increased compared with that in untreated patients (Figure 8, F, I, and O). In cervical spinal cord, the total percentage of SMN-positive cells and the percentage of low-SMN-positive cells were significantly increased in treated compared with untreated SMA spinal cords (Figure 8, D,
G, and M), while thoracic cord showed a trend (Figure 8, E, H, and $\mathrm{N})$. At all spinal levels assessed, the percentages of SMN-positive cells in treated patients were either not significantly different or were higher relative to those in unaffected controls (Figure 8, J-O). Some SMN-positive cells were detected in frontal and temporal cortex in 2 treated cases (Supplemental Figure 5).

\section{Discussion}

In order to assess SMN expression in human tissues, we enacted a multistate and decade-long program of expedited autopsy tis- 
sue collection from an unprecedented number of SMA and agematched non-SMA individuals. The standardized autopsy protocol implemented for this study involved the collection and processing of tissues as quickly after death as possible in order to maximize quality for protein and RNA studies. The majority of our tissues (68\%) had a PMI of less than 12 hours, and nearly all (97\%) had a PMI of less than 24 hours. Several studies document the integrity of biochemical assessments done within these time frames (2733), and formal statistical consideration of PMI in our study indicated that it did not significantly affect outcomes. The importance of establishing SMN levels in relevant human tissues has become particularly urgent in SMA, as breakthrough SMN-inducing therapeutics have now advanced to clinical trials or commercial use (11). However, efforts to optimize their potential clinical benefit are constrained by limited data describing normal, SMA disease baseline or present dosage regimen-achieved drug-induced SMN transcript and protein levels in the CNS, which cannot be assessed in living patients. The data reported here have important implications for optimization of therapeutics for SMA patients, but also highlight the importance of implementing such expedited autopsy programs to collect high-quality human CNS tissues in patients with other neurological diseases being targeted with novel gene targeting therapeutics $(34,35)$.

SMN protein levels decrease during development. Our data reveal a substantial, 6.5-fold decline of normal SMN protein expression in the human spinal cord between fetal and postnatal stages. Measured by 3 different methods, SMN protein levels show a broad range in spinal cord, cortex, and muscle tissues isolated during the second and third trimester, but become restricted at low levels postnatally, particularly after 3 months, in both controls and SMA subjects. These data are consistent with a limited number of prior studies describing perinatal decline of SMN protein expression in human $(36,37)$ and mouse $(20,38-42)$ spinal cord and muscle via Western blot and immunohistochemistry. However, quantitative assessment of SMN in these studies, particularly in human spinal cord, has often been limited due to the small number of samples. Evidence of elevated SMN expression in blood of young children compared with adults has also been reported $(20,43)$.

Elevated SMN levels early in development suggest a particular need for SMN in the CNS during gestational and neonatal stages of motor neuron development. Consistent with this notion is the observation that when SMN is reduced gestationally or within the first 2 weeks of life in mice, an SMA phenotype is reliably induced (44), but depletion of SMN in adult mice does not result in overt SMA-like phenotypes $(45,46)$. Further, while disease phenotypes in severe SMA mouse models can be dramatically reversed using genetic or pharmacological interventions delivered embryonically or early postnatally, benefits are modest when given after the first week $(12,15,47)$. The specific cellular consequences of reduced SMN levels at specific stages of human development are not known, but the observation that treatment instituted before 6 weeks of life is associated with markedly improved efficacy is consistent with a high need for SMN neonatally in humans. Our data particularly highlight a window encompassing the last trimester of gestation and the first 3 months after birth, when SMN protein may decrease quite rapidly. The nature of an autopsy study precludes longitudinal measures to determine whether the timing of this decrease varies subject to subject, but the range of values seen in the second and third trimesters and early postnatal cases suggests that this may be the case. Variations in this timing could be an important determinant of clinical response to SMN induction even when given very early. Our cohort included a limited number of SMA subjects collected during gestation $(n=1)$ and the first 3 months of life $(n=3)$, but SMN levels were reduced in SMA spinal cord samples compared with those in age-matched controls during these intervals (4-fold prenatal, 6-fold early postnatal). This contrasts with the nonstatistically significant differences in SMN protein levels between control and SMA samples aged greater than 3 months. Together with limited data from prior studies (8, $36,48,49)$, this suggests that magnitude of SMN protein reduction in SMA may be most marked during fetal and neonatal stages.

An important technical caveat of our data is that quantitative SMN protein measures are possible only on total spinal cord lysates and are normalized to total protein amount (or in the case of Western blot, normalized to specific endogenous control proteins). Previous studies have suggested a developmental decline in SMN expression specifically in motor neurons in rodents and humans by immunohistochemistry $(37,39,40,48)$. Unfortunately, lack of appropriately fixed fetal spinal cord tissues precluded our ability to perform immunohistochemistry of SMN in fetal compared with adult motor neurons in this study.

Mechanisms regulating SMN expression during development. Developmental changes in SMN protein levels were accompanied by more modest reductions of SMN1 and SMN2 transcript levels, but no change in SMN2 mRNA exon 7 splicing patterns. Prior studies suggest that $S M N$ gene promoter activity may decrease during development and neuronal differentiation $(4,50,51)$, and we have previously shown that epigenetic changes at the $S M N$ gene promoters, including those mediated by histone acetylation (41) and a long noncoding RNA, SMN-AS1 (21), repress the SMN promoter during development. Because of the high-sequence similarity between the SMN1 and SMN2 promoters, it has been difficult to determine whether or not the genes undergo parallel regulation in humans, but luciferase reporter assays identify an approximately 2-fold higher promoter activity of SMN1 compared with SMN2 (4). Interestingly, while SMN2-FL decreased perinatally, SMN1-FL did not significantly decrease until late postnatal ages. This earlier decrease of SMN2 transcriptional activity could further contribute to earlier developmental reductions in SMN protein in SMA patients compared with controls. While multivariable quantile regression analysis demonstrated that both SMN1-FL and SMN2-FL contribute to SMN protein expression, they (along with PMI) may not fully account for the changes in SMN protein expression observed with increased age. Along with the observation that prenatal diaphragm expresses relatively high SMN1-FL, but low SMN protein, levels, this suggests that mechanisms other than transcriptional activity or splicing, such as translational efficiency and/or protein stability, may contribute to SMN protein expression and that variations in these other molecular regulators could also account for variations of therapeutic response in patients. Further investigation is needed to dissect such mechanisms, as this could lead to therapeutic strategies that could enhance SMN expression in combination with splicing modification (21).

Implications for timing of treatment. Recent clinical trials of SMN-inducing drugs nusinersen and scAAV9-SMN demonstrate a 
range of clinical efficacy, with the time of treatment initiation playing a critical role in the magnitude of clinical response $(16,17,52)$. In a phase III trial of nusinersen, symptomatic infants with 2 copies of SMN2 dosed starting at an average age of 5.4 months showed reduced mortality, and $51 \%$ demonstrated improvements in motor function compared with $0 \%$ of infants in the control group, with $8 \%$ sitting independently at study completion (16). In a phase I trial of onasemnogene abeparvovec-xioi, 12 SMA type I infants were dosed with high doses at an average age of 3.4 months, with $92 \%$ achieving sitting and $16 \%$ walking (17). In data from presymptomatic SMA infants (15 SMN2 copy number = 2, 10 SMN2 copy number $=3$ ), treatment with nusinersen was initiated at less than 6 weeks of life, and after a median of 2.9 years of treatment, $100 \%$ of children sat and $88 \%$ walked independently $(52,53)$. Together, these trials powerfully illustrate that a delay of SMN induction of weeks or months can substantially reduce achievement of motor milestones. The data reported here provide a possible explanation for both the general benefit of earlier treatment and the individual differences in response. SMN levels were variably low in control subjects in the third trimester, but consistently low by 3 months of age. If a normal developmental decrease in SMN expression is a factor in the initiation of motor neuron dysfunction in those with SMA, earlier initiation of SMN enhancement during this period could thus be more effective. Recently, the American Advisory Committee on Heritable Disorders in Newborns and Children in the US recommended SMA as a condition meeting the criteria for nationwide newborn screening. As of March 2019, 18 states have begun to develop and implement screening programs. The data presented here emphasize the urgency of this effort as well as the importance of mobilizing infrastructure to provide SMN induction treatment as soon after birth as possible (54). Moreover, these results raise the possibility that SMN induction prior to birth will be required for optimal patient outcomes at least in some patients.

Topographical variations of SMN induction with nusinersen treatment. SMA is characterized by a stereotypical pattern of muscle weakness, with proximal muscles more affected than distal, limbs more affected than face, and chest wall more affected than diaphragm. We did not identify striking regional differences in baseline SMN protein expression between spinal cord segments that might account for these regional variations. Interestingly, prenatal diaphragm samples expressed approximately 2-fold lower SMN protein compared with prenatal iliopsoas or spinal cord. This could indicate that resistance to disease in tissues such as the diaphragm is conferred by an overall lower requirement for SMN protein during gestation. Alternatively, intrinsic differences in the rate of development of different tissues might determine the timing of SMN decrements. Of note, the diaphragm is known to mature more quickly than other muscle groups because of the need to breathe immediately upon birth (55) and consequently might experience an earlier decrease in SMN expression.

Nusinersen does not cross the blood-brain barrier and thus must be delivered by intermittent lumbar intrathecal injection. Limited data obtained during autopsies of 3 severely affected infants (ages 12.8, 5.2, and 11.4 months old) dosed with nusinersen by lumbar injection in an early clinical trial (26) demonstrated higher drug concentrations in caudal compared with rostral tissues (lumbar cord: 26.6, 31.8, and $19.4 \mu \mathrm{g} / \mathrm{g}$ vs. brainstem: 13.8, 8.13, and $3.68 \mu \mathrm{g} / \mathrm{g}$ ). A previous study of an ASO targeting huntingtin mRNA following lumbar intrathecal injection in rhesus monkeys showed a similar rostral-to-caudal gradient of drug concentrations (56). Although our autopsy cases may be biased toward those with poor clinical outcomes, our data also demonstrate regional variation in ASO distribution. In SMA patients receiving more than 1 dose of nusinersen, higher ASO concentrations in lumbar/ sacral, thoracic, and cervical spinal levels were also associated with an increase in SMN2-FL transcript levels. Importantly, the current recommended dosage of nusinersen is $12 \mathrm{mg}$ in a volume of $5 \mathrm{~mL}$, regardless of patient age and size. CSF volume increases from $8 \mathrm{~mL}$ in neonates to $150 \mathrm{~mL}$ by 5 years of age $(57,58)$. Results from a study modeling nusinersen pharmacokinetics in CSF from treated SMA patients suggest that patients over 2 years of age have reduced ASO distribution in the CSF relative to patients under 2 years of age (59). The 2 treated patients with the most robust SMN induction in our cohort were 1 and 2 years old at the time of death. Two patients that were treated with more than one dose of nusinersen and demonstrated less SMN induction were 4 years and 13.5 years old at time of death. Whether larger volumes or higher amounts of nusinersen would improve ASO distribution in older patients is an important area for future investigation.

Despite increases in $S M N 2-F L$ transcript levels, particularly in caudal spinal cord regions, no increase in SMN protein levels in whole spinal cord tissue was observed in any treated case. Our IHC revealed cellular uptake of ASO by a small percentage of cells in spinal cord and cortex tissues, with this uptake most evident in neurons, in part due to their larger cell bodies. Quantification of SMN IHC staining intensity in treated versus untreated samples was technically challenging, but an increase in SMN expression in motor neurons from treated subjects was observed, as reported previously (26). Limited increase in SMN protein staining was observed in the majority of cells in the spinal cord. Given that motor neurons make up a very small percentage of the total cells in the spinal cord, the lack of whole-tissue protein increase is not surprising given these IHC results. Additional work is needed to fully characterize nusinersen effects in different cell populations, but it is possible that the apparent limited targeting of nusinersen to different CNS cell types also limits its efficacy in some patients, particularly as an increasing body of literature suggests potential contributions of cell types other than motor neurons in disease pathogenesis (60).

The results from this study have important implications beyond the treatment of SMA patients. ASOs are being developed or are in clinical trials for numerous neurological diseases, including Huntington's disease and amyotrophic lateral sclerosis. Efficacy will require efficient delivery to neurons in spinal and brain regions as well as other cell types, such as glia, implicated in disease pathogenesis. Our data from a limited number of subjects highlight the difficulty in achieving high ASO levels in rostral CNS regions after intrathecal delivery and the cell-type variability in ASO uptake. Further work to optimize both ASO delivery and distribution will likely ultimately benefit patients with different neurological diseases.

\section{Methods}

Human samples. Tissue samples were dissected at autopsy and immediately flash-frozen in liquid nitrogen or fixed in $4 \%$ paraformaldehyde for 24 hours at $4^{\circ} \mathrm{C}$, washed $3 \times 10$ minutes in PBS, and stored in 
either PBS or 30\% sucrose. Tissues used for histology were cut using a human brain protocol on a Sakura Tissue Tek tissue processor. After embedding, slides were cut at 4 microns, air dried overnight, then dried at $60^{\circ} \mathrm{C}$ for 1 hour.

HTRF assay. Tissue samples were homogenized on the TissueLyzer II (QIAGEN) in RIPA buffer (50 mM Tris-HCl, pH 7.4; $150 \mathrm{mM} \mathrm{NaCl}$; $1 \% \mathrm{NP}-40$ [v/v]; 0.5\% sodium deoxycholate [w/v]; 0.1\% SDS [w/v]) containing a cocktail of protease inhibitors (Roche) at a tissue weight to RIPA buffer volume of $50 \mathrm{mg} / \mathrm{mL}$. The samples were then centrifuged for 20 minutes at $14,000 \mathrm{~g}$ at $4^{\circ} \mathrm{C}$. The homogenates were transferred to a 96-well plate and were diluted in RIPA buffer to approximately $1 \mathrm{mg} / \mathrm{mL}$ for SMN HTRF assay (Cisbio) and $0.5 \mathrm{mg} / \mathrm{mL}$ for total soluble protein measurement using the BCA Protein Assay (Pierce). Samples were run in duplicate and averaged. For the SMN HTRF assay, $35 \mu \mathrm{L}$ of tissue homogenate was transferred to a 384-well plate containing $5 \mu \mathrm{L}$ of the antibody solution (1:100 dilution of anti-SMN d2 [acceptor] and anti-SMN cryptate [donor]). The plate was incubated for 20 hours in the dark at room temperature. Fluorescence was measured at 665 $\mathrm{nm}$ and $620 \mathrm{~nm}$ on an EnVision Plate Reader (PerkinElmer). The data were processed according to the Cisbio protocol (ref. 19 and https:// www.cisbio.com/content/signal-treatment-and-analysis). SMN protein signal $\left(\Delta \mathrm{F}=\left([665 \mathrm{~nm} / 620 \mathrm{~nm}]_{\text {sample }}-[665 \mathrm{~nm} / 620 \mathrm{~nm}]_{\text {background }}\right]\right) /$ $\left.[665 \mathrm{~nm} / 620 \mathrm{~nm}]_{\text {background }}\right)$ was normalized to total soluble protein.

SMN immunohistochemistry. Slides were stained with rabbit monoclonal anti-SMN (Abcam, catalog ab108424) antibody on a Ventana Ultra staining system. SMN slides received Ventana's CC1-heat induced antigen retrieval (Ventana, 950-500) for 92 minutes. The primary antibody was diluted with Discovery Antibody Diluent (Ventana, 760-108) and incubated for 1 hour at $37^{\circ} \mathrm{C}$. The antibodies were detected with biotin-labeled goat anti-rabbit secondary antibody (Jackson ImmunoResearch Laboratories, catalog 111-005-003). The secondary antibody was labeled with the Chromomap DAB Kit (Ventana, 760-124). Images were scanned on a Hamamatsu $\mathrm{S} 360$ scanner at $\times 40$ resolution.

SMN IHC quantification. Tissues showing morphological signs of autolysis or unspecific dark background were excluded from further quantification. SMN expression in glial and neuronal cells of the CNS was quantified using a customer-developed App with Visiopharm image analysis software (VIS 2019.02.1.6005; visiopharm.com). CNS regions of interest (ROI) in digitalized images were manually applied and, for the spinal cord, color coded to separate lumbar, thoracic, and cervical cord. Tissue folds, artificially darkened tissue edges, and blood vessels were excluded from quantification. The app detected all cells within the ROI, quantified their area and intensity, and binned them into 4 categories based on staining intensity (either negative for SMN staining or low, medium, or high SMN staining) based on user-taught parameters. A ratio of negative-, low-, medium-, or highexpressing SMN cells per total cells in the ROI was calculated.

ASO immunohistochemistry. Slides were stained with rabbit polyclonal ASO (Ionis Pharmaceuticals) (56) antibody on a Ventana Ultra Staining System. ASO slides were treated enzymatically with Trypsin (MilliporeSigma, T8003). The slides were then blocked with Endogenous Biotin Blocking Kit (Ventana, 760-050) and normal goat serum (Jackson ImmunoResearch, 005-000-121). The primary antibody was diluted with Discovery Antibody Diluent (Ventana, 760-108) and incubated for 1 hour at $37^{\circ} \mathrm{C}$. The antibodies were detected with biotin-labeled goat anti-rabbit secondary antibody (Jackson ImmunoResearch, catalog 111-005-003). The secondary antibody was labeled with the DABMap Kit (Ventana, 760-124). Images were scanned on a Hamamatsu $\mathrm{S} 360$ scanner at $\times 40$ resolution.

ASO IHC quantification. Tissues showing morphological signs of autolysis were excluded from further quantification. ASO uptake in glial, neuronal, and endothelial cells of CNS was scored semiquantitatively by a board-certified pathologist. Scores ranged from 0 to 3 , with $0=$ no uptake, 1 = minimal uptake, 2 = good uptake, and 3 = strong uptake.

ASO concentration measurement. Human autopsy samples were analyzed using a hybridization ELISA method. Briefly, human samples and monkey brain for a calibration curve and monkey brains for quality control (QC) were minced, weighed, homogenized in buffer, and extracted by liquid-liquid extraction using phenol chloroform. The aqueous was then dried down under vacuum and reconstituted in 500 $\mu \mathrm{L}$ of control human plasma. Samples were then diluted into range with control human plasma and run with a hybridization-based ELISA method. A Molecular Devices Gemini XPS lnstrument was used for the final hybridization ELISA (HELISA) analysis. The calibration curve range of the assay for parent was $0.0200-1.50 \mu \mathrm{g} / \mathrm{g}$, with the low end of the range defining the lower limit of quantification (LLOQ) in 50 mg of control cynomolgus monkey brain (for all brain and spinal cord sections). All samples were quantified within the quantitative range of the assay $(0.0200$ to $1.50 \mu \mathrm{g} / \mathrm{g})$. The percentage of coefficient of variation (CV) between duplicate wells on the plate was less than $20 \%$ for all curves, QCs, and samples. All samples were stored at $-70^{\circ} \mathrm{C}$ to $10^{\circ} \mathrm{C}$ upon receipt. Human lumbar, thoracic, and cervical spinal cord samples as well as frontal or temporal cortex, cerebellum, pons, or midbrain were all analyzed, as available for each patient, under the same conditions used for monkey brain tissue for calibrators and QC samples.

RNA isolation and RT-qPCR analysis. Tissues were homogenized in TRIzol reagent (Thermo Fisher) using Lysing Matrix D Tubes (MP Biomedicals) in a Fastprep-24 instrument (MP Biomedicals) using 3 rounds of homogenization at a velocity of $6 \mathrm{~m} / \mathrm{s}$ with 5 -minute intervals on ice to avoid heating of the samples. Samples were subsequently mixed with $70 \%(\mathrm{v} / \mathrm{v})$ ethanol and transferred into RNeasy columns (QIAGEN). Total RNA was cleaned up and eluted in nuclease-free water using the manufacturer's protocol. Total RNA concentration was determined using a Nanodrop-2000 spectrophotometer (Thermo Fisher) with minimal required 260/280 absorbance ratios of 1.8. Equal amounts of RNA were converted into cDNA using the High-Capacity cDNA Reverse Transcription Kit (Thermo Fisher) per the manufacturer's recommendations. RT-qPCR was performed with the 7900HT Real-Time PCR System (Thermo Fisher) using TaqMan Universal PCR Master Mix (Thermo Fisher) and custom FAM-based TaqMan assays (Thermo Fisher; see Supplemental Table 2 for sequences) using the manufacturer's recommendations. The expression levels of $S M N-\triangle 5$ and 8 different housekeeping genes were evaluated using custom RT-qPCR SYBR Green primers (Supplemental Table 2) and SYBR Green Universal PCR Master Mix (Thermo Fisher) per the manufacturer's protocol. The stability of the housekeeping genes was evaluated by GeNorm analysis using qbase + software (Biogazelle), and SMN levels were normalized to the levels of the 2 or 3 most stable housekeeping genes (61). Each sample was run in triplicate, and the average normalized $S M N$ data were calibrated to a unique sample set to 1 . All data are expressed as calibrated normalized relative quantities, as previously described (61).

Absolute real-time RT-PCR. Real-time RT-PCR was performed following the manufacturer's instructions for the Express One-Step Superscript qRT-PCR Kit (Thermo Fisher Scientific) by Asuragen, as previous- 
ly described (26). The final concentrations of primers and probes were 125 and $62.5 \mathrm{nM}$, respectively. RT-PCR reactions of calibration standards and autopsy RNA samples were performed using an ABI 7900HT Real-Time PCR Instrument. The thermal profile was as follows: $50^{\circ} \mathrm{C}$ for 15 minutes, $90^{\circ} \mathrm{C}$ for 2 minutes, and 40 cycles of $95^{\circ} \mathrm{C}$ for 15 seconds and $60^{\circ} \mathrm{C}$ for 1 minute. Optical data from all runs were analyzed using the automatic baseline setting and automatic threshold. The threshold cycle value for each sample was converted to a copy number by extrapolation to the calibration curve. For each sample, measurements were performed in triplicate, and the average is reported. The percentage of full-length SMN2 transcript was calculated as a percentage of the sum of SMN2 transcripts that include or exclude exon 7. The absolute real-time RT-PCR method was evaluated by Asuragen for upper and lower limits of detection and quantification, inter- and intraassay precision and accuracy, dilution linearity, and specificity.

Statistics. Statistical analyses for analysis of SMN RNA and protein analysis based on age were performed in STATA 14.2. Median values and IQR for each age group of each tissue were generated. $P$ values of pairwise comparison of medians by age group were calculated using Wilcoxon's rank-sum test. For comparisons between tissues, a Kruskal-Wallis test was used to determine whether differences in RNA or protein expression existed between tissues in each age group. $P$ values were generated for pair-wise comparisons of medians using Wilcoxon's rank-sum test. Bonferroni's correction was applied to adjust for multiple comparisons between control age groups. Three pairwise comparisons were made in each analysis resulting in an adjusted $\alpha$ level of 0.0167 (0.05/3). $P$ values before multiple comparisons are represented in figures to highlight biologically significant differences that may be lost after adjustment for multiple comparisons due to relatively small sample size. Comparisons between control and SMA within each age group were independent and thus not adjusted for multiple comparisons. Univariable quantile regression analysis was used to test for effects of PMI on SMN protein and RNA as well as the effects of SMN1-FL and SMN2-FL RNA on SMN protein. Based on the scatterplot of PMI versus SMN protein expression, PMI was modeled using a quadratic term to best model the distribution of the data. Multivariable quantile regression analysis was used to adjust for effects of PMI, SMN1-FL, and/or SMN2-FL on SMN protein changes with age.

For nusinersen-treated cases (Figures 7 and 8), statistical analyses were performed using Prism version 7.03 (GraphPad). The level of significance was set to 0.05. For 2-way ANOVA, variation between groups was assumed equal (standard in GraphPad Prism), followed by correction for multiple comparisons using Tukey's multiple comparisons test for all experimental groups compared. Data related to nusinersen treatment (Figures 7 and 8) are represented as mean \pm SEM, except with individual data points overlaid onto the charts. Simple linear regression was performed using Prism version 7.03 (GraphPad).

Additional methods for electrochemiluminescence assay (20), Western blot (21), and SMN1 and SMN2 gene copy number analysis (62) are available in the Supplemental Methods.
Study approval. Study protocols were approved by the Office of Human Subjects Research Institutional Review Board at Johns Hopkins University (protocol IRB NA_00035399) and the Institutional Review Board at the University of Utah School of Medicine. Written, informed consent for autopsy was received from patients or their parents prior to autopsy. Other samples were obtained from the NIH NeuroBioBank. Some cases were collected in coordination with the Living Legacy Foundation. Deidentified samples were stored with only age, sex, SMA status, postmortem time, and tissue type.

\section{Author contributions}

DMR, CDY, NN, SP, TOC, and CJS designed the research studies. DMR, CDY, VG, AD, SKK, DAN, JM, SJT, PGZ, PJS, and IW conducted experiments. DMR, CDY, VG, AD, SKK, DAN, JM, SJT, PGZ, PJS, and IW acquired data. DMR, CDY, SKK, and NG analyzed data. DV, CLH, TWP, and NN provided reagents and samples for the study. DMR, CDY, and CJS wrote the manuscript. DMR, CDY, VG, AD, SKK, DAN, JM, SJT, PGZ, TWP, PJS, DV, CLH, IW, NG, KJS, FR, CFB, NN, SP, TOC, and CJS critically revised the manuscript.

\section{Acknowledgments}

We would like to express our profound gratitude for the generosity and altruism of donors and their families, whose gifts of organs and tissues serve an integral role in advancing medical research and education, as well as our colleagues who referred patients, including Kathryn J. Swoboda, John Brandsema, Gihan Tennekoon, Samiah Al-Zaidy, Richard Shell, Nancy Kuntz, and Christine DiDonato, among others. We also thank the numerous pathologists with whom we have worked, including Jody Hooper, who leads the rapid autopsy program at Johns Hopkins, and Brian Harding at Children's Hospital of Philadelphia. Additionally, we would like to thank Kathleen Smart at Children's National Hospital for aid in program coordination. We also acknowledge the support of The Living Legacy Foundation of Maryland, the organization that works with donor families to honor their loved ones through the donation of organs and tissue for transplantation, education, and research. Some human tissues were received from the NIH NeuroBioBank at the University of Maryland (Baltimore, Maryland, USA). This work was supported by funding to CJS from the SMA Foundation, SMART, the NIH (R01-NS096770, R01-NS062869), Ionis, and PTC Therapeutics. KJS receives grant support from the NIH. Biogen provided support for absolute SMN2 mRNA quantitation and absolute real-time RT-PCR.

Address correspondence to: Charlotte J. Sumner, Departments of Neurology and Neuroscience, 855 North Wolfe Street, John G. Rangos Sr. Building, Room 248, Baltimore, Maryland 21205, USA. Phone: 410.502.6085; Email: csumner1@jhmi.edu.
1. Verhaart IEC, et al. Prevalence, incidence and carrier frequency of 5q-linked spinal muscular atrophy - a literature review. Orphanet J Rare Dis. 2017;12(1):124.

2. Lefebvre $S$, et al. Identification and characterization of a spinal muscular atrophy-determining gene. Cell. 1995;80(1):155-165.
3. Lorson CL, Hahnen E, Androphy EJ, Wirth B. A single nucleotide in the SMN gene regulates splicing and is responsible for spinal muscular atrophy. Proc Natl Acad Sci U S A. 1999;96(11):6307-6311.

4. Monani UR, McPherson JD, Burghes AH. Promoter analysis of the human centromeric and telomeric survival motor neuron genes (SMNC and SMNT). Biochim Biophys Acta. 1999;1445(3):330-336.

5. Cartegni L, Krainer AR. Disruption of an SF2/ ASF-dependent exonic splicing enhancer in SMN2 causes spinal muscular atrophy in the absence of SMN1. Nat Genet. 2002;30(4):377-384. 
6. Kashima T, Manley JL. A negative element in SMN2 exon 7 inhibits splicing in spinal muscular atrophy. Nat Genet. 2003;34(4):460-463.

7. Fischer U, Liu Q, Dreyfuss G. The SMN-SIP1 complex has an essential role in spliceosomal snRNP biogenesis. Cell. 1997;90(6):1023-1029.

8. Lefebvre S, et al. Correlation between severity and SMN protein level in spinal muscular atrophy. Nat Genet. 1997;16(3):265-269.

9. Parsons DW, McAndrew PE, Iannaccone ST, Mendell JR, Burghes AH, Prior TW. Intragenic telSMN mutations: frequency, distribution, evidence of a founder effect, and modification of the spinal muscular atrophy phenotype by cenSMN copy number. Am J Hum Genet. 1998;63(6):1712-1723.

10. Prior TW, Swoboda KJ, Scott HD, Hejmanowski AQ. Homozygous SMN1 deletions in unaffected family members and modification of the phenotype by SMN2. Am J Med Genet A. 2004;130A(3):307-310.

11. Sumner CJ, Crawford TO. Two breakthrough gene-targeted treatments for spinal muscular atrophy: challenges remain. J Clin Invest. 2018;128(8):3219-3227.

12. Hua Y, et al. Peripheral SMN restoration is essential for long-term rescue of a severe spinal muscular atrophy mouse model. Nature. 2011;478(7367):123-126.

13. Naryshkin NA, et al. Motor neuron disease. SMN2 splicing modifiers improve motor function and longevity in mice with spinal muscular atrophy. Science. 2014;345(6197):688-693.

14. Palacino J, et al. SMN2 splice modulators enhance U1-pre-mRNA association and rescue SMA mice. Nat Chem Biol. 2015;11(7):511-517.

15. Foust KD, et al. Rescue of the spinal muscular atrophy phenotype in a mouse model by early postnatal delivery of SMN. Nat Biotechnol. 2010;28(3):271-274.

16. Finkel RS, et al. Nusinersen versus sham control in infantile-onset spinal muscular atrophy. $N$ Engl JMed. 2017;377(18):1723-1732.

17. Mendell JR, et al. Single-dose gene-replacement therapy for spinal muscular atrophy. $N$ EnglJ Med. 2017;377(18):1713-1722.

18. Mercuri E, et al. Nusinersen versus sham control in later-onset spinal muscular atrophy. $\mathrm{N}$ Engl $J$ Med. 2018;378(7):625-635.

19. Degorce F, Card A, Soh S, Trinquet E, Knapik GP, Xie B. HTRF: A technology tailored for drug discovery - a review of theoretical aspects and recent applications. Curr Chem Genomics. 2009;3:22-32

20. Zaworski P, et al. SMN protein can be reliably measured in whole blood with an electrochemiluminescence (ECL) immunoassay: implications for clinical trials. PLOS ONE. 2016;11(3):e0150640.

21. d'Ydewalle $\mathrm{C}$, et al. The antisense transcript SMN-AS1 regulates SMN expression and is a novel therapeutic target for spinal muscular atrophy. Neuron. 2017;93(1):66-79.

22. Gennarelli M, et al. Survival motor neuron gene transcript analysis in muscles from spinal muscular atrophy patients. Biochem Biophys Res Commun. 1995;213(1):342-348.

23. Singh NN, Seo J, Rahn SJ, Singh RN. A multiexon-skipping detection assay reveals surprising diversity of splice isoforms of spinal muscular atrophy genes. PLOS ONE. 2012;7(11):e49595.

24. Martinez TL, et al. Survival motor neuron protein in motor neurons determines synaptic integrity in spinal muscular atrophy. J Neurosci. 2012;32(25):8703-8715.

25. Bricceno KV, et al. Survival motor neuron protein deficiency impairs myotube formation by altering myogenic gene expression and focal adhesion dynamics. Hum Mol Genet. 2014;23(18):4745-4757.

26. Finkel RS, et al. Treatment of infantile-onset spinal muscular atrophy with nusinersen: a phase 2, open-label, dose-escalation study. Lancet. 2016;388(10063):3017-3026.

27. Blair JA, et al. Individual case analysis of postmortem interval time on brain tissue preservation. PLOS ONE. 2016;11(3):e0151615.

28. Stan AD, et al. Human postmortem tissue: what quality markers matter? Brain Res. 2006;1123(1):1-11.

29. Li X, Greenwood AF, Powers R, Jope RS. Effects of postmortem interval, age, and Alzheimer's disease on G-proteins in human brain. Neurobiol Aging. 1996;17(1):115-122.

30. Ferrer I, et al. Brain protein preservation largely depends on the postmortem storage temperature: implications for study of proteins in human neurologic diseases and management of brain banks: a BrainNet Europe Study. J Neuropathol Exp Neurol. 2007;66(1):35-46.

31. Ervin JF, et al. Postmortem delay has minimal effect on brain RNA integrity. J Neuropathol Exp Neurol. 2007;66(12):1093-1099.

32. Liu X, Brun A. Synaptophysin immunoreactivity is stable $36 \mathrm{~h}$ postmortem. Dementia. 1995;6(4):211-217.

33. Hynd MR, Lewohl JM, Scott HL, Dodd PR. Biochemical and molecular studies using human autopsy brain tissue. J Neurochem. 2003;85(3):543-562.

34. Schoch KM, Miller TM. Antisense oligonucleotides: translation from mouse models to human neurodegenerative diseases. Neuron. 2017;94(6):1056-1070.

35. Ly CV, Miller TM. Emerging antisense oligonucleotide and viral therapies for amyotrophic lateral sclerosis. Curr Opin Neurol. 2018;31(5):648-654.

36. Burlet $\mathrm{P}$, et al. The distribution of SMN protein complex in human fetal tissues and its alteration in spinal muscular atrophy. Hum Mol Genet. 1998; 7(12):1927-1933.

37. Giavazzi A, Setola V, Simonati A, Battaglia G. Neuronal-specific roles of the survival motor neuron protein: evidence from survival motor neuron expression patterns in the developing human central nervous system. J Neuropathol Exp Neurol. 2006;65(3):267-277.

38. La Bella V, Cisterni C, Salaün D, Pettmann B. Survival motor neuron (SMN) protein in rat is expressed as different molecular forms and is developmentally regulated. Eur J Neurosci. 1998;10(9):2913-2923.

39. Battaglia G, Princivalle A, Forti F, Lizier C, Zeviani M. Expression of the SMN gene, the spinal muscular atrophy determining gene, in the mammalian central nervous system. Hum Mol Genet. 1997;6(11):1961-1971.
40. Pagliardini S, et al. Subcellular localization and axonal transport of the survival motor neuron (SMN) protein in the developing rat spinal cord. Hum Mol Genet. 2000;9(1):47-56.

41. Kernochan LE, et al. The role of histone acetylation in SMN gene expression. Hum Mol Genet. 2005;14(9):1171-1182.

42. Groen EJN, et al. Temporal and tissue-specific variability of SMN protein levels in mouse models of spinal muscular atrophy. Hum Mol Genet. 2018;27(16):2851-2862.

43. Wadman RI, et al. A comparative study of SMN protein and mRNA in blood and fibroblasts in patients with spinal muscular atrophy and healthy controls. PLOS ONE. 2016;11(11):e0167087.

44. Schrank B, et al. Inactivation of the survival motor neuron gene, a candidate gene for human spinal muscular atrophy, leads to massive cell death in early mouse embryos. Proc Natl Acad Sci US A. 1997;94(18):9920-9925.

45. Sahashi K, et al. TSUNAMI: an antisense method to phenocopy splicing-associated diseases in animals. Genes Dev. 2012;26(16):1874-1884.

46. Kariya S, et al. Requirement of enhanced Survival Motoneuron protein imposed during neuromuscular junction maturation. J Clin Invest. 2014;124(2):785-800.

47. Lutz CM, et al. Postsymptomatic restoration of SMN rescues the disease phenotype in a mouse model of severe spinal muscular atrophy. J Clin Invest. 2011;121(8):3029-3041.

48. Kletzl H, et al. SMN protein levels before and after treatment with risdiplam (RG7916) in patients with type 1 to 3 SMA compared to healthy subjects. Paper presented at: 23 rd International Annual Congress of the World Muscle Society; October 2-6, 2018; Mendoza, Argentina.

49. Soler-Botija C, Cuscó I, Caselles L, López E, Baiget M, Tizzano EF. Implication of fetal SMN2 expression in type ISMA pathogenesis: protection or pathological gain of function? J Neuropathol Exp Neurol. 2005;64(3):215-223.

50. Echaniz-Laguna A, Miniou P, Bartholdi D, Melki J. The promoters of the survival motor neuron gene (SMN) and its copy (SMNc) share common regulatory elements. Am J Hum Genet. 1999;64(5):1365-1370.

51. Rouget R, et al. Characterization of the survival motor neuron (SMN) promoter provides evidence for complex combinatorial regulation in undifferentiated and differentiated P19 cells. Biochem J. 2005;385(Pt 2):433-443.

52. Swoboda KJ, et al. Nusinersen in infants who initiate treatment in a pre-symptomatic stage of spinal muscular atrophy (SMA): interim efficacy and safety results from the phase 2 NURTURE study. Paper presented at: 23 rd International Annual Congress of the World Muscle Society; October 2-6, 2018; Mendoza, Argentina.

53. De Vivo DC, et al. Nusinersen initiated in infants during the presymptomatic stage of spinal muscular atrophy: Interim efficacy and safety results from the Phase 2 NURTURE study [published online ahead of print September 12, 2019]. Neuromuscul Disord. https://doi.org/10.1016/j. nmd.2019.09.007.

54. Glascock J, et al. Treatment algorithm for 
infants diagnosed with spinal muscular atrophy through newborn screening. J Neuromuscul Dis. 2018;5(2):145-158.

55. Mantilla CB, Sieck GC. Key aspects of phrenic motoneuron and diaphragm muscle development during the perinatal period. J Appl Physiol. 2008;104(6):1818-1827.

56. Kordasiewicz HB, et al. Sustained therapeutic reversal of Huntington's disease by transient repression of huntingtin synthesis. Neuron. 2012;74(6):1031-1044.

57. Rochette A, et al. Cerebrospinal fluid volume in neonates undergoing spinal anaesthesia: a descriptive magnetic resonance imaging study. Br J Anaesth. 2016;117(2):214-219.

58. Johanson CE, Duncan JA, Klinge PM, Brinker T, Stopa EG, Silverberg GD. Multiplicity of cerebrospinal fluid functions: New challenges in health and disease. Cerebrospinal Fluid Res. 2008;5:10.

59. Luu KT, Norris DA, Gunawan R, Henry S, Geary $\mathrm{R}$, Wang Y. Population pharmacokinetics of nusinersen in the cerebral spinal fluid and plasma of pediatric patients with spinal muscular atrophy following intrathecal administrations.
JClin Pharmacol. 2017;57(8):1031-1041.

60. Bowerman $\mathrm{M}$, et al. Therapeutic strategies for spinal muscular atrophy: SMN and beyond. Dis Model Mech. 2017;10(8):943-954.

61. Vandesompele J, et al. Accurate normalization of real-time quantitative RT-PCR data by geometric averaging of multiple internal control genes. Genome Biol. 2002;3(7):RESEARCH0034.

62. McAndrew PE, et al. Identification of proximal spinal muscular atrophy carriers and patients by analysis of SMNT and SMNC gene copy number. Am J Hum Genet. 1997;60(6):1411-1422. 